\title{
On Cooperative Solutions of a Generalized Assignment Game: Limit Theorems to the Set of Competitive Equilibria
}

\author{
JORDI MASSÓ (Corresponding author) \\ Universitat Autònoma de Barcelona and Barcelona GSE \\ Departament d'Economia i d'Història Econòmica \\ Edifici B, UAB. 08193, Bellaterra (Barcelona), Spain \\ Phone: +34935812370. E-mail: jordi.masso@uab.es \\ Alejandro Neme \\ Universidad Nacional de San Luis and CONICET \\ Instituto de Matemática Aplicada de San Luis \\ Ejército de los Andes 950. 5700, San Luis, Argentina \\ Phone: +542652422803. E-mail: aneme@unsl.edu.ar
}

First version: February 2010

This version: May 2013

\begin{abstract}
We study two cooperative solutions of a market with indivisible goods modeled as a generalized assignment game: Set-wise stability and Core. We establish that the Set-wise stable set is contained in the Core and contains the non-empty set of competitive equilibrium payoffs. We then state and prove three limit results for replicated markets. First, the Set-wise stable set of a two-fold replicated market already coincides with the set of competitive equilibrium payoffs. Second, the sequence of Cores of replicated markets converges to the set of competitive equilibrium payoffs when the number of replicas tends to infinity. Third, for any number of replicas there is a market with a Core payoff that is not a competitive equilibrium payoff.
\end{abstract}

Journal of Economic Literature Classification Numbers: C78; D78.

Keywords: Assignment game; Core; Set-wise stability; Competitive equilibrium. 


\section{Introduction}

We study two cooperative solutions for a class of markets with indivisible goods modeled as generalized assignment games. Shapley and Shubik (1972) defined an assignment game as a market where each seller owns one indivisible object and each buyer, who wants to buy at most one object, has valuations over all objects. An assignment is a description of deliveries of objects from sellers to buyers and a price vector is a list of prices, one for each object. A competitive equilibrium of a market is a price vector and a feasible assignment at which each seller maximizes revenues, each buyer maximizes net valuations, and markets clear. Shapley and Shubik (1972) showed that the set of competitive equilibria is non-empty, competitive equilibrium assignments are optimal (the first welfare theorem holds), any optimal assignment is part of a competitive equilibrium with any of the competitive equilibrium price vectors (a strong version of the second welfare theorem holds without requiring any redistribution of the initial endowments), and the set of competitive equilibrium payoffs coincides with the Core of a naturally associated TU-game (no enlargement or replica of the market is required for their coincidence).

We consider a generalized assignment game representing a market with a given number of indivisible units of different goods, where sellers may own different units of each of the goods and buyers, who may want to buy several units of different goods up to an exogenous total amount, have constant marginal valuations of each good. Jaume, Massó, and Neme (2012) extend Shapley and Shubik (1972)'s results for this generalized assignment game. In particular, they show that the set of competitive equilibria is non-empty, it is the Cartesian product of the set of competitive equilibrium price vectors and the set of optimal assignments, the set of competitive equilibrium price vectors has a lattice structure with the natural partial order of vectors $\geq$ "to be larger or equal than", and this lattice structure is partly translated in a dual way to the sets of buyers and sellers' utilities that are attainable at competitive equilibria.

In this paper we study two different cooperative solutions for this class of markets and their relationship with the set of competitive equilibrium payoffs. The two solutions differ on how a coalition of buyers and sellers can block a proposed payoff vector. Given an assignment and a coalition of buyers and sellers, some of them may be buying or selling some units of some goods to sellers or buyers outside the coalition. The notion of the Core corresponds to the notion of blocking that requires that all members of the coalition have to break all exchanges performed with all agents outside the coalition and buy or sell only with 
members within the coalition. In contrast, the concept of Set-wise stability corresponds to the notion of blocking that admits that members of the coalition may completely or partly keep their exchanges performed with non-members.

The Set-wise stability notion is closer to the already well established notion of stability applied to ordinal many-to-one matching models. For instance, Roth and Sotomayor (1990) analyze this model and its applications to college admission problems and to labor markets for medical interns assuming that a hospital in a blocking coalition can maintain its relationships with interns outside the coalition. There is no reason to expect that the hospital, in order to make an offer to a doctor in the blocking coalition, will have to cancel the contracts it has with other doctors it has been already assigned to.

Since Set-wise blocking is easier than Core-wise blocking, the Set-wise stable set is a subset of the Core. We show here that the non-empty set of competitive equilibrium payoffs is contained in the Set-wise stable set. Hence, the Set-wise stable set as well as the Core are non-empty. Moreover, we exhibit a simple market showing that these inclusions may be strict.

The main contribution of the paper is to answer affirmatively the following question. Do the Core and the Set-wise stable set converge to the set of competitive equilibrium payoffs when the market becomes large? The question is relevant because competitive equilibrium requires price-taking behavior which only makes sense when individual quantity decisions are perceived by each agent as being negligible. To create a setting where price-taking behavior is meaningful we follow the well established tradition in Economics to enlarge the environment by replicating the market. We first show that the Set-wise stable set already coincides with the set of competitive equilibrium payoffs for a two-fold replicated market (Theorem 1). We also show that the Core converges to the set of competitive equilibrium payoffs when the number of replica tends to infinity (Theorem 2). Finally, we show that for any number of replicas there is a market with a Core payoff that is not a competitive equilibrium payoff (Theorem 3). Thus, the notion of Set-wise stability is much closer (not only in terms of set-wise inclusion) to competitive equilibrium than the notion of Core.

There are many other papers that recently have studied the relationship between the set of competitive equilibrium payoffs and alternative cooperative solutions in many-to-one or many-to-many generalizations of Shapley and Shubik (1972)'s assignment game. Sotomayor (1992 and 1999a) study a many-to-many assignment game with two finite and disjoint sets of agents. Each agent from each side can form a maximal number of partnerships with the agents from the other side. Each partnership generates a total payoff that may be shared 
by its two members. Observe that in this extension partnerships are binary; specifically, if a buyer and a seller form a partnership they can exchange just one indivisible unit of the good held by the seller. Sotomayor (1992) proves that all pair-wise stable assignments are optimal and Sotomayor (1999a) shows that the set of pair-wise stable payoffs has a complete and dual lattice structure. Sotomayor (1999b) proposes the notion of Set-wise stability for the former model and shows that the pair-wise stable set (that may be empty) is a subset of the Core. Camiña (2006) studies a market with one seller, that owns a given number of (potentially) different objects, and several buyers who want to buy at most one object. She shows that the Core and the Set-wise stable set coincide, the set of competitive equilibrium payoffs is non-empty and it is a subset of the Core. Moreover, she shows that the Core has a complete lattice structure with the partial order coming from comparing buyers' payoff vectors with the partial order $\geq$ and this structure is not dual.

Sotomayor (2007) studies a generalized assignment game similar to ours but with two important differences: (i) sellers only own units of a unique good and each good is only owned by a particular seller and (ii) buyers may want to buy several units but partnerships are also binary because buyers are not interested in buying more than one unit from each seller. She shows that the set of competitive equilibrium payoffs is a non-empty, complete and dual lattice. Sotomayor (2009 and 2011) extends Sotomayor (1992 and 1999a) and consider a time-sharing assignment game where both buyers and sellers own a fixed amount of a divisible good (labor time) and to form a partnership a buyer and a seller have to agree to contribute each with the same amount of labor time and to share, in a particular proportion, the amount of money that is proportionally obtained from the jointly contributed amount of labor time. Sotomayor (2009) studies different solution concepts for different kinds of coalitional interactions. In particular, she shows the inclusion relationships that hold among the non-empty sets of competitive equilibrium payoffs, the Core, the Set-wise stable set, the Strong stable set and the set of dual allocations. Moreover, she also shows that some of these sets have a lattice structure. Sotomayor (2011) analyses the relationship between the competitive equilibrium solution and a cooperative notion similar to Set-wise stability that differs from ours because agreements with non-members are rigid since they are nullified once any of its terms is changed. Moreover, Sotomayor (2011) studies the algebraic structure of these sets. Klaus and Walzl (2009) study several notions of Setwise stability for the ordinal many-to-many matching model with contracts under different preference restrictions.

Milgrom (2009) introduces and studies the space of assignment messages to investigate 
(and solve) the difficulty that agents face when reporting their "types" (or valuations of goods, or sets of goods) in some mechanism design settings. The model is very general and contains as particular cases multi-unit auctions (with substitutable goods), exchange economies, and integer assignment games. Milgrom (2009) focuses on the study of the non-emptiness of the set of competitive equilibrium prices and its lattice structure but he does not analyze any cooperative solution. Jaume, Massó, and Neme (2012) study using linear programming the same model than the present one but they only focus on the study of the Cartesian product and lattice structures of the set of competitive equilibria and the corresponding sets of agents' utilities.

The paper is organized as follows. In Section 2 we define a market. In Section 3, and closely following Jaume, Massó, and Neme (2012), we define a competitive equilibrium of a market. In Section 4 we first present the notion of Set-wise stability and show that the Setwise stable set contains the non-empty set of competitive equilibrium payoffs. We define, for any positive integer $\rho$, a $\rho$-fold replica of a market and show in Theorem 1 that the Setwise stable set of a two-fold replicated market already coincides with the set of competitive equilibrium payoffs. We then present the notion of Core and show that it contains the set of Set-wise stable payoffs and in Theorem 2 we show that the limit of the sequence of the Cores of replicated markets coincides with the set of competitive equilibrium payoffs when the number of replicas tends to infinity. Finally, in Theorem 3 we show that for any number of replicas there is a market with a Core payoff that is not a competitive equilibrium payoff. In Section 5 we discuss our main result and finish with final remarks. An Appendix at the end of the paper collects the proofs that have been omitted in the main text.

\section{Preliminaries}

A generalized assignment game (a market) consists of three finite and disjoint sets: the set $\mathcal{B}=\{1, \ldots, B\}$ of buyers, the set $\mathcal{G}=\{1, \ldots, G\}$ of goods, and the set $\mathcal{S}=\{1, \ldots, S\}$ of sellers. We denote a generic buyer by $i$, a generic good by $j$, and a generic seller by $k$. Buyers have a constant marginal valuation of each good. Let $v_{i j} \geq 0$ be the monetary valuation that buyer $i$ assigns to each unit of good $j$; namely, $v_{i j}$ is the maximum price that buyer $i$ is willing to pay for each unit of good $j$. Denote by $V=\left(v_{i j}\right)_{(i, j) \in \mathcal{B} \times \mathcal{G}}$ the matrix of valuations. We assume that buyer $i \in \mathcal{B}$ can buy at most $d_{i} \in \mathbb{Z}_{+} \backslash\{0\}$ units in total, where $\mathbb{Z}_{+}$is the set of non-negative integers. The strictly positive integer $d_{i}$ should be interpreted as a capacity constraint due to limits on i's ability for storage, transport, etc. Denote by 
$d=\left(d_{i}\right)_{i \in \mathcal{B}}$ the vector of maximal demands. Each seller $k \in \mathcal{S}$ has $q_{j k} \in \mathbb{Z}_{+}$indivisible units of each good $j \in \mathcal{G}$. Denote by $Q=\left(q_{j k}\right)_{(j, k) \in \mathcal{G} \times \mathcal{S}}$ the capacity matrix. We assume that there is a strictly amount of each good; namely,

$$
\text { for each } j \in \mathcal{G} \text { there exists } k \in \mathcal{S} \text { such that } q_{j k}>0 \text {. }
$$

Let $r_{j k} \geq 0$ be the monetary valuation that seller $k$ assigns to each unit of good $j$; that is, $r_{j k}$ is the reservation (or minimum) price that seller $k$ is willing to accept for each unit of good $j$. Denote by $R=\left(r_{j k}\right)_{(j, k) \in \mathcal{G} \times \mathcal{S}}$ the matrix of reservation prices.

A market $M$ is a 7 -tuple $(\mathcal{B}, \mathcal{G}, \mathcal{S}, V, d, R, Q)$ satisfying condition (1). Shapley and Shubik (1972)'s (one-to-one) assignment game is a special case of a market where each buyer can buy at most one unit, there is only one unit of each good, and each seller only owns one unit of one of the goods; i.e., $d_{i}=1$ for all $i \in \mathcal{B}, G=S$, and for all $(j, k) \in \mathcal{G} \times \mathcal{S}$, $q_{j k}=1$ if $j=k$ and $q_{j k}=0$ if $j \neq k$.

An assignment for market $M=(\mathcal{B}, \mathcal{G}, \mathcal{S}, V, d, R, Q)$ is a three-dimensional integer matrix (i.e., a $3^{r d}$-order tensor) $A=\left(A_{i j k}\right)_{(i, j, k) \in \mathcal{B} \times \mathcal{G} \times \mathcal{S}} \in \mathbb{Z}_{+}^{B \times G \times S}$ describing a collection of deliveries of units of the goods from buyers to sellers. Each $A_{i j k}$ should be interpreted as "buyer $i$ receives $A_{i j k}$ units of good $j$ from seller $k$." We often omit the sets to which the subscripts belong to and write, for instance, $\sum_{i j k} A_{i j k}$ and $\sum_{i} A_{i j k}$ instead of $\sum_{(i, j, k) \in \mathcal{B} \times \mathcal{G} \times \mathcal{S}} A_{i j k}$ and $\sum_{i \in \mathcal{B}} A_{i j k}$, respectively.

The assignment $A$ is feasible for market $M=(\mathcal{B}, \mathcal{G}, \mathcal{S}, V, d, R, Q)$ if each buyer $i$ buys at most $d_{i}$ units and each seller $k$ sells at most $q_{j k}$ units of each good $j$. We are only interested on the following set of feasible assignments

$\left\{A \in \mathbb{Z}_{+}^{B \times G \times S} \mid \sum_{j k} A_{i j k} \leq d_{i}\right.$ for all $i \in \mathcal{B}$ and $\sum_{i} A_{i j k} \leq q_{j k}$ for all $\left.(j, k) \in \mathcal{G} \times \mathcal{S}\right\}$

For each $(i, j, k) \in \mathcal{B} \times \mathcal{G} \times \mathcal{S}$, let

$$
\tau_{i j k}= \begin{cases}v_{i j}-r_{j k} & \text { if } q_{j k}>0 \\ 0 & \text { if } q_{j k}=0\end{cases}
$$

be the per unit gain from trade of good $j$ between buyer $i$ and seller $k$. If seller $k$ does not have any unit of good $j$ the per unit gain from trade of good $j$ with all buyers is equal to zero. The total gain from trade of market $M=(\mathcal{B}, \mathcal{G}, \mathcal{S}, V, d, R, Q)$ at assignment $A$ is

$$
T^{M}(A)=\sum_{i j k} \tau_{i j k} \cdot A_{i j k}
$$


Definition 1 A feasible assignment $A$ is optimal for market $M=(\mathcal{B}, \mathcal{G}, \mathcal{S}, V, d, R, Q)$ if, for any feasible assignment $A^{\prime}, T^{M}(A) \geq T^{M}\left(A^{\prime}\right)$.

Let $\mathcal{F}$ be the set of all optimal assignments for market $M=(\mathcal{B}, \mathcal{G}, \mathcal{S}, V, d, R, Q)$. The set $\mathcal{F}$ is always non-empty. ${ }^{1}$ Denote by $T^{M}$ the total gain from trade of market $M$ at any optimal assignment.

\section{Competitive Equilibrium}

\subsection{Definitions and Preliminaries}

We define a competitive equilibrium of market $M=(\mathcal{B}, \mathcal{G}, \mathcal{S}, V, d, R, Q)$ by following Jaume, Massó and Neme (2012). Assume buyers and sellers trade through competitive markets. That is, there is a unique market (and its corresponding unique price) for each of the goods and buyers and sellers are price-takers. Given a price vector $p=\left(p_{j}\right)_{j \in \mathcal{G}} \in \mathbb{R}_{+}^{G}$ sellers supply units of the goods (up to their capacity) in order to maximize revenues at $p$ and buyers demand units of the goods (up to their maximal demands) in order to maximize the total net valuation at $p$.

Supply of seller $k$ : For each price vector $p=\left(p_{j}\right)_{j \in \mathcal{G}} \in \mathbb{R}_{+}^{G}$, seller $k$ supplies of every good $j$ any feasible amount that maximizes revenues; namely,

$$
\mathcal{S}_{j k}\left(p_{j}\right)= \begin{cases}\left\{q_{j k}\right\} & \text { if } p_{j}>r_{j k} \\ \left\{0,1, \ldots, q_{j k}\right\} & \text { if } p_{j}=r_{j k} \\ \{0\} & \text { if } p_{j}<r_{j k}\end{cases}
$$

To define the demands of buyers we need the following notation. Let $p \in \mathbb{R}_{+}^{G}$ be given and consider buyer $i$. Let

$$
\nabla_{i}^{>}(p)=\left\{j \in \mathcal{G} \mid v_{i j}-p_{j}=\max _{j^{\prime} \in \mathcal{G}}\left\{v_{i j^{\prime}}-p_{j^{\prime}}\right\}>0\right\}
$$

be the set of goods that give to buyer $i$ the maximum (and strictly positive) net valuation at $p$. Obviously, for some $p$, the set $\nabla_{i}^{>}(p)$ may be empty. Let

$$
\nabla_{i}^{\geq}(p)=\left\{j \in \mathcal{G} \mid v_{i j}-p_{j}=\max _{j^{\prime} \in \mathcal{G}}\left\{v_{i j^{\prime}}-p_{j^{\prime}}\right\} \geq 0\right\}
$$

\footnotetext{
${ }^{1}$ See Milgrom (2009) for a proof of this statement in a more general model. See Jaume, Massó and Neme (2012) for a proof of the statement using only linear programming arguments in the same model as the one studied here.
} 
be the set of goods that give to buyer $i$ the maximum (and non-negative) net valuation at $p$. Obviously, for some $p$, the set $\nabla_{i}^{\geq}(p)$ may also be empty. Obviously, for all $p \in \mathbb{R}_{+}^{G}$ and all $i \in \mathcal{B}$,

$$
\nabla_{i}^{>}(p) \subseteq \nabla_{i}^{\geq}(p)
$$

Demand of buyer $i$ : For each price vector $p=\left(p_{j}\right)_{j \in \mathcal{G}} \in \mathbb{R}_{+}^{G}$, buyer $i$ demands any feasible amounts of the goods that maximize the net valuations at $p$; namely,

$$
\begin{aligned}
D_{i}(p)=\left\{\alpha=\left(\alpha_{j k}\right)_{(j, k) \in \mathcal{G} \times \mathcal{S}} \in \mathbb{Z}^{\mathcal{G} \times \mathcal{S}} \mid\right. & \text { (D.a) } \alpha_{j k} \geq 0 \text { for all }(j, k) \in \mathcal{G} \times \mathcal{S}, \\
& \text { (D.b) } \sum_{j k} \alpha_{j k} \leq d_{i}, \\
& \text { (D.c) } \nabla_{i}^{>}(p) \neq \emptyset \Longrightarrow \sum_{j k} \alpha_{j k}=d_{i}, \text { and } \\
& \text { (D.d) } \left.\sum_{k} \alpha_{j k}>0 \Longrightarrow j \in \nabla_{i}^{\geq}(p)\right\} .
\end{aligned}
$$

Thus, $D_{i}(p)$ describes the set of all trades that maximize the net valuation of buyer $i$ at $p$. Observe that the set of trades described by each element in the set $D_{i}(p)$ give the same net valuation to buyer $i$; i.e., $i$ is indifferent among all trade plans $\alpha \in D_{i}(p)$.

Let $A$ be an assignment and let $i$ be a buyer. We denote by $A(i)=\left(A(i)_{j k}\right)_{(j, k) \in \mathcal{G} \times \mathcal{S}}$ the element in $\mathbb{Z}_{+}^{\mathcal{G} \times \mathcal{S}}$ such that, for all $(j, k) \in \mathcal{G} \times \mathcal{S}, A(i)_{j k}=A_{i j k}$.

Definition $2 A$ competitive equilibrium of market $M=(\mathcal{B}, \mathcal{G}, \mathcal{S}, V, d, R, Q)$ is a pair $(p, A) \in \mathbb{R}_{+}^{G} \times \mathbb{Z}_{+}^{B \times G \times S}$ such that $A$ is a feasible assignment and:

(E.D) for each buyer $i \in \mathcal{B}, A(i) \in D_{i}(p)$;

(E.S) for each good $j \in \mathcal{G}$ and each seller $k \in \mathcal{S}, \sum_{i} A_{i j k} \in \mathcal{S}_{j k}\left(p_{j}\right)$.

The vector $p \in \mathbb{R}_{+}^{G}$ is a competitive equilibrium price of market $M=(\mathcal{B}, \mathcal{G}, \mathcal{S}, V, d, R, Q)$ if there exists a feasible assignment $A$ such that $(p, A)$ is a competitive equilibrium of market $M$. Let $\mathcal{P}$ be the set of competitive equilibrium prices of market $M$. The set $\mathcal{P}$ is always non-empty. ${ }^{2}$ For further reference, we state this fact without proof as a remark below.

Remark 1 The set of competitive equilibrium prices of any market is non-empty.

Moreover, by Proposition 4 in Jaume, Massó, and Neme (2012), the set of competitive equilibria has a Cartesian product structure. We also state this fact without proof as a remark below.

Remark 2 Let $M=(\mathcal{B}, \mathcal{G}, \mathcal{S}, V, d, R, Q)$ be a market. Then, $(p, A)$ is a competitive equilibrium of $M$ if and only if $p \in \mathcal{P}$ and $A \in \mathcal{F}$.

\footnotetext{
${ }^{2}$ For the proof of this statement in a more general model see Milgrom (2009), and for a proof in our setting using only linear programming see Jaume, Massó and Neme (2009).
} 


\subsection{The Set of Competitive Equilibrium Payoffs}

Let $p \in \mathbb{R}_{+}^{G}$ be a price vector and $A$ a feasible assignment of market $M=(\mathcal{B}, \mathcal{G}, \mathcal{S}, V, d, R, Q)$. We define the utility of buyer $i \in \mathcal{B}$ at the pair $(p, A)$ as the total net gain obtained by $i$ from his exchanges specified by $A$ at price $p$. We denote it by $u_{i}(p, A)$; namely,

$$
u_{i}(p, A)=\sum_{j k}\left(v_{i j}-p_{j}\right) \cdot A_{i j k} .
$$

We define the utility of seller $k \in \mathcal{S}$ at the pair $(p, A)$ as the total net gain obtained by $k$ from his exchanges specified by $A$ at price $p$. We denote it by $w_{k}(p, A)$; namely,

$$
w_{k}(p, A)=\sum_{i j}\left(p_{j}-r_{j k}\right) \cdot A_{i j k}
$$

Given $(p, A)$, denote by $u(p, A)=\left(u_{i}(p, A)\right)_{i \in \mathcal{B}}$ and $w(p, A)=\left(w_{k}(p, A)\right)_{k \in \mathcal{S}}$ the vector of buyers and sellers' utilities at $(p, A)$, respectively. Let

$$
\mathcal{C} \mathcal{E}=\left\{(u, w) \in \mathbb{R}^{B} \times \mathbb{R}^{S} \mid \text { there exists }(p, A) \in \mathcal{P} \times \mathcal{F} \text { s.t. }(u, w)=(u(p, A), w(p, A))\right\}
$$

be the set of competitive equilibrium payoffs of market $M=(\mathcal{B}, \mathcal{G}, \mathcal{S}, V, d, R, Q)$. However, competitive equilibrium payoffs are independent of the particular optimal assignment. To see that, define the mappings of per-unit gains $\gamma(\cdot): \mathbb{R}_{+}^{G} \rightarrow \mathbb{R}^{B}$ and $\pi(\cdot): \mathbb{R}_{+}^{G} \rightarrow \mathbb{R}^{G \times S}$ as follows. Let $p \in \mathbb{R}_{+}^{G}$ be given. For each $i \in \mathcal{B}$, define

$$
\gamma_{i}(p)= \begin{cases}v_{i j}-p_{j} & \text { if there exists } j \in \nabla_{i}^{>}(p) \\ 0 & \text { otherwise, }\end{cases}
$$

and for each $(j, k) \in \mathcal{G} \times \mathcal{S}$, define

$$
\pi_{j k}(p)= \begin{cases}p_{j}-r_{j k} & \text { if } p_{j}-r_{j k}>0 \\ 0 & \text { otherwise. }\end{cases}
$$

The number $\gamma_{i}(p)$ is the gain obtained by buyer $i$ from each unit that he wants to buy at $p$ (if any) and the number $\pi_{j k}(p)$ is the profit obtained by seller $k$ from each unit of good $j$ that he wants to sell at $p$ (if any).

Let $p \in \mathcal{P}$ be a competitive equilibrium price of market $M=(\mathcal{B}, \mathcal{G}, \mathcal{S}, V, d, R, Q)$ and let $(\gamma(p), \pi(p))$ be its associated per unit gains. Define $(u(p), w(p)) \in \mathbb{R}^{B} \times \mathbb{R}^{S}$ by setting

$$
\begin{aligned}
u_{i}(p) & =d_{i} \cdot \gamma_{i}(p) \text { for all } i \in \mathcal{B} \text { and } \\
w_{k}(p) & =\sum_{j \in \mathcal{G}} q_{j k} \cdot \pi_{j k}(p) \text { for all } k \in \mathcal{S} .
\end{aligned}
$$


By Lemma 6 in Jaume, Massó, and Neme (2012), the set of competitive equilibrium payoffs of market $M$ can also be written as

$$
\mathcal{C E}=\left\{(u, w) \in \mathbb{R}^{B} \times \mathbb{R}^{S} \mid \text { there exists } p \in \mathcal{P} \text { such that }(u, w)=(u(p), w(p))\right\}
$$

that is, the set of competitive equilibrium payoffs of market $M$ can be described without explicitly referring to any particular optimal assignment because, for all $A \in \mathcal{F}, u_{i}(p, A)=$ $u_{i}(p)$ for all $i \in \mathcal{B}$ and $w_{k}(p, A)=w_{k}(p)$ for all $k \in \mathcal{S}$.

\section{Cooperative Solutions}

In the next two subsections we study two alternative cooperative solutions for market $M$. They differ on how a coalition (a subset) of agents can block a proposal of how to distribute among all agents the total gain from trade obtained at any optimal assignment. The Core assumes that members of a blocking coalition can only form partnerships among themselves and have to break all former partnerships with non-members. Set-wise stability allows members of a blocking coalition to keep or reduce their former exchanges with members outside the blocking coalition. Thus, Set-wise blocking is easier than Core-wise blocking. It seems to us that Set-wise stability is also a more reasonable solution for this class of markets. Our results will indicate from two points of view that Set-wise stability is closer to the set of competitive equilibrium payoffs than the Core is: (i) (set inclusion) closer and (ii) the set of Set-wise stable payoffs and the set of competitive equilibrium payoffs already coincide in a two-fold replicated market.

\subsection{Set-wise Stability}

The notion of Core-blocking requires that all members of the blocking coalition have to give up all previous exchange agreements with non-members. However this may be too drastic because, in some circumstances, it is reasonable to let members of the blocking coalition to keep some (or all) previous exchanges with members outside the blocking coalition. This stronger notion of blocking gives rise to the notion of Set-wise stability. ${ }^{3}$ Let $M=$

\footnotetext{
${ }^{3}$ Sotomayor (1999b) defines and studies this concept for a generalization of Shapley and Shubik (1972)'s assignment game. See also Sotomayor (2007, 2009, and 2011) for an analysis of Set-wise stability in her time-sharing assignment games. Klaus and Walzl (2009) have studied Strong and Set-wise stability for an ordinal many-to-many matchings model with contracts under different preference restrictions. Our notion
} 
$(\mathcal{B}, \mathcal{G}, \mathcal{S}, V, d, R, Q)$ be a market and let $\mathcal{C} \subseteq \mathcal{B} \cup \mathcal{S}$ be a coalition. Denote the subsets of buyers and sellers in $\mathcal{C}$ by $\mathcal{B}^{\mathcal{C}}=\mathcal{C} \cap \mathcal{B}$ and $\mathcal{S}^{\mathcal{C}}=\mathcal{C} \cap \mathcal{S}$, respectively.

Definition 3 Let $M=(\mathcal{B}, \mathcal{G}, \mathcal{S}, V, d, R, Q)$ be a market and $\mathcal{C}$ be a coalition. A feasible assignment $\widehat{A}$ for market $M$ is $\mathrm{SW}$-compatible with $\mathcal{C}$ if there exists an optimal assignment $A \in \mathcal{F}$ such that:

(i) For every $i \in \mathcal{B}^{\mathcal{C}}, \widehat{A}_{i j k}>0$ implies that either $k \in \mathcal{S}^{\mathcal{C}}$ or else $\widehat{A}_{i j k} \leq A_{i j k}$.

(ii) For every $k \in \mathcal{S}^{\mathcal{C}}, \widehat{A}_{i j k}>0$ implies that either $i \in \mathcal{B}^{\mathcal{C}}$ or else $\widehat{A}_{i j k} \leq A_{i j k}$.

(iii) For every $i \notin \mathcal{B}^{\mathcal{C}}$ and $k \notin \mathcal{S}^{\mathcal{C}}, \widehat{A}_{i j k}=0$ for every $j \in \mathcal{G}$.

We want to emphasize that the definition of $S W$-compatibility allows to reallocate in any way the amount of the goods exchanged between a buyer and a seller if they both belong to the coalition but only to keep or decrease their exchanges if one is a member of the coalition and the other is not.

Let $M=(\mathcal{B}, \mathcal{G}, \mathcal{S}, V, d, R, Q)$ be a market. A three-dimensional matrix $\Gamma=\left(\Gamma_{i j k}\right)_{(i, j, k) \in \mathcal{B} \times \mathcal{G} \times \mathcal{S}}$ is a distribution matrix if for all $(i, j, k) \in \mathcal{B} \times \mathcal{G} \times \mathcal{S}$ such that $v_{i j} \geq r_{j k}$, it holds that $v_{i j} \geq \Gamma_{i j k} \geq r_{j k}$. Let $\Gamma$ be a distribution matrix and assume $v_{i j} \geq r_{j k}$ for some $(i, j, k) \in \mathcal{B} \times \mathcal{G} \times \mathcal{S}$. Then, $\Gamma_{i j k}$ describes a way of how buyer $i$ and seller $k$ could split the gain $v_{i j}-r_{j k}$ that they would obtain from trading one unit of good $j$ : buyer $i$ receives $v_{i j}-\Gamma_{i j k}$ and seller $k$ receives $\Gamma_{i j k}-r_{j k}$. If $v_{i j}<r_{j k}$ then the value $\Gamma_{i j k}$ will be irrelevant because $i$ and $k$ do not trade good $j$ at any optimal assignment. Observe that a distribution matrix is not necessarily anonymous because a buyer can obtain different per unit gains from buying good $j$ from two different sellers, and vice versa.

Definition 4 A payoff $(u, w) \in \mathbb{R}^{B} \times \mathbb{R}^{S}$ for market $M=(\mathcal{B}, \mathcal{G}, \mathcal{S}, V, d, R, Q)$ is not SW-blocked if there exists a distribution matrix $\Gamma=\left(\Gamma_{i j k}\right)_{(i, j, k) \in \mathcal{B} \times \mathcal{G} \times \mathcal{S}}$ such that for every coalition $\mathcal{C} \subset \mathcal{B} \cup \mathcal{S}$ and every feasible assignment $\widehat{A}$ that is $S W$-compatible with $\mathcal{C}$, we have

of Set-wise stability as well as Klaus and Walzl (2009)'s stability notions are natural extensions of the concept first defined by Sotomayor (1999b). The main difference between our notion and Klaus and Walzl (2009)'s notions is that ours applies to a transferable utility setting while theirs apply to an ordinal setting. This is important when checking the profitability of a deviating coalition; for the transferable utility setting utilities of all members in the coalition are added while in the ordinal setting all members of the blocking coalition have to receive (in ordinal terms) a better (for Strong stability) and individually rational (for Set-wise stability) set of contracts. 
that

$$
\begin{aligned}
\sum_{i \in \mathcal{B}^{\mathcal{C}}} u_{i}+\sum_{k \in \mathcal{S}^{\mathcal{C}}} w_{k} & \geq \sum_{(i, j, k) \in \mathcal{B}^{\mathcal{C}} \times \mathcal{G} \times \mathcal{S}^{\mathcal{C}}} \tau_{i j k} \cdot \widehat{A}_{i j k}+\sum_{(i, j, k) \in \mathcal{B}^{\mathcal{C}} \times \mathcal{G} \times\left(\mathcal{S}^{\mathcal{C}}\right)^{c}}\left(v_{i j}-\Gamma_{i j k}\right) \cdot \widehat{A}_{i j k} \\
& +\sum_{(i, j, k) \in\left(\mathcal{B}^{\mathcal{C}}\right)^{c} \times \mathcal{G} \times \mathcal{S}^{\mathcal{C}}}\left(\Gamma_{i j k}-r_{j k}\right) \cdot \widehat{A}_{i j k} . \\
& \equiv \phi^{M}(\mathcal{C}, \widehat{A}, \Gamma) .
\end{aligned}
$$

A payoff $(u, w) \in \mathbb{R}^{B} \times \mathbb{R}^{S}$ is not $S W$-blocked if there is a set of exchanges between buyers and sellers (the optimal assignment $A$ in Definition 3) and a set of agreements on how to share the per unit gains from trade (the distribution matrix $\Gamma$ ) such that no coalition of agents, independently of the agreements they have with non-members, can jointly obtain a strictly higher payoff by reassigning their exchanges among themselves and by keeping or reducing their exchanges with non-members. Observe that by letting $\mathcal{C}=\mathcal{B} \cup \mathcal{S}, \sum_{i \in \mathcal{B}} u_{i}+\sum_{k \in \mathcal{S}} w_{k}=T^{M}$. That is, agents can optimally achieve $(u, w)$ in a way that is immune to deviating coalitions. Finally, a payoff vector is Set-wise stable if it can not be $S W$-blocked.

Definition 5 A payoff $(u, w) \in \mathbb{R}^{B} \times \mathbb{R}^{S}$ for market $M=(\mathcal{B}, \mathcal{G}, \mathcal{S}, V, d, R, Q)$ is Set-wise stable if it is not $S W$-blocked.

Denote by $\mathcal{S W}$ the set of Set-wise stable payoffs of market $M=(\mathcal{B}, \mathcal{G}, \mathcal{S}, V, d, R, Q)$. When we want to emphasize market $M$ we write $\mathcal{S} \mathcal{W}^{M}$. The set of competitive equilibrium payoffs is contained in the set of Set-wise stable payoffs.

Proposition 1 Let $p \in \mathcal{P}$ be a competitive equilibrium price of market $M=(\mathcal{B}, \mathcal{G}, \mathcal{S}, V, d, R, Q)$. Then, $(u(p), w(p)) \in \mathcal{S} \mathcal{W}$.

Proof See the Appendix.

The idea of the proof is as follows. Assume $p \in \mathcal{P}$ and $(u(p), w(p)) \notin \mathcal{S W}$. This means that every distribution matrix $\Gamma$ has a $S W$-block. In particular, we consider one of the $S W$-blocks of the anonymous distribution matrix $p$ (i.e., for all $j \in \mathcal{G}, p_{j}=\Gamma_{i j k}=\Gamma_{i^{\prime} j k^{\prime}}$ for all $i, i^{\prime}, k$ and $k^{\prime}$. Hence, there exist a coalition $\mathcal{C}$ and a feasible assignment $\widehat{A}$,

\footnotetext{
${ }^{4}$ Given a set $X$ we denote its complementary set by $X^{c}$. The reader should not be confused with this notation when the set $X$ is either $\mathcal{B}^{\mathcal{C}}$ or $\mathcal{S}^{\mathcal{C}}$, whose complements are denoted by $\left(\mathcal{B}^{\mathcal{C}}\right)^{c}$ and $\left(\mathcal{S}^{\mathcal{C}}\right)^{c}$, respectively.
} 
$S W$-compatible with $\mathcal{C}$, such that $\mathcal{C}$ can obtain a strictly larger payoff. The key step of the proof is to construct from $\widehat{A}$ a reduced market $\bar{M}$ by keeping fixed and excluding from $\bar{M}$ the exchanges specified by $\widehat{A}$ between a member of $\mathcal{C}$ and a non member of $\mathcal{C}$. Namely, the maximal demand $d_{i}$ of every buyer $i$ in the blocking coalition is reduced by the number of units that $i$ buys to sellers outside the coalition. The maximal capacity $q_{j k}$ of each good $j$ owned by every seller $k$ in the blocking coalition is reduced by the number of units of good $j$ that $k$ sells to buyers outside the coalition. Then, although tedious, it only remains to be shown that the Strong Duality theorem of Linear Programming is violated (see the Appendix at the end of the paper), a contradiction. ${ }^{5}$

Remark 1 and Proposition 1 above say that

$$
\varnothing \neq \mathcal{C} \mathcal{E} \subset \mathcal{S W}
$$

Example 1 below shows that the inclusion in (11) may be strict because there exist markets with a payoff $(u, w) \in \mathcal{S} \mathcal{W} \backslash \mathcal{C E}$.

Example 1 Let $M=(\mathcal{B}, \mathcal{S}, \mathcal{G}, V, d, R, Q)$ be a market where $\mathcal{B}=\left\{b_{1}, b_{2}\right\}, \mathcal{G}=\left\{g_{1}, g_{2}\right\}$, $\mathcal{S}=\left\{s_{1}\right\}$ (i.e., $B=G=2$ and $\left.S=1\right), V=\left(\begin{array}{ll}8 & 4 \\ 8 & 1\end{array}\right), d=(2,3), R=(4,2)$, and $Q=(4,1)$. The unique optimal assignment of market $M$ is $A=\left(\begin{array}{ll}1 & 1 \\ 3 & 0\end{array}\right)$ and

$$
\begin{aligned}
T^{M}(A) & =\left(v_{11}-r_{11}\right) \cdot A_{111}+\left(v_{12}-r_{21}\right) \cdot A_{121}+\left(v_{21}-r_{11}\right) \cdot A_{211}+\left(v_{22}-r_{21}\right) \cdot A_{221} \\
& =(8-4) \cdot 1+(4-2) \cdot 1+(8-4) \cdot 3+(1-2) \cdot 0 \\
& =18
\end{aligned}
$$

It is easy to see that the set of competitive equilibrium prices of market $M$ is $\mathcal{P}=\left\{\left(p_{1}, p_{2}\right) \in\right.$ $\mathbb{R}_{+}^{2} \mid 2 \leq p_{2} \leq 4$ and $\left.p_{1}=p_{2}+4\right\}$. For every $p \in \mathcal{P}$, the per-unit gains are

$$
\begin{aligned}
\gamma_{1}(p) & =8-p_{1}=4-p_{2}, \\
\gamma_{2}(p) & =8-p_{1}, \\
\pi_{11}(p) & =p_{1}-4, \text { and } \\
\pi_{21}(p) & =p_{2}-2 .
\end{aligned}
$$

\footnotetext{
${ }^{5}$ Or equivalently, we reach the conclusion that coalition $\mathcal{C}$ in market $\bar{M}$ Core-blocks $(u(p), w(p))$, a contradiction since, as we will see in the next subsection, competitive equilibrium payoffs can not be Core-blocked.
} 
Moreover $u_{1}(p)=2 \cdot \gamma_{1}(p)=16-2 p_{1}, u_{2}(p)=3 \cdot \gamma_{2}(p)=24-3 p_{1}$ and $w_{1}(p)=4 \cdot \pi_{11}(p)+$ $1 \cdot \pi_{21}(p)=5 \cdot p_{1}-22$. The set of competitive equilibrium payoffs is

$$
\mathcal{C E}=\left\{\left(u_{1}, u_{2}, w_{1}\right) \in \mathbb{R}_{+}^{3} \mid u_{1}=16-2 p_{1}, u_{2}=24-3 p_{1}, w_{1}=5 p_{1}-22 \text { and } 6 \leq p_{1} \leq 8\right\} .
$$

Consider the vector $\left(u_{1}, u_{2}, w_{1}\right)=\left(\frac{11}{3}, 5, \frac{28}{3}\right)$. It is immediate to see that $\left(\frac{11}{3}, 5, \frac{28}{3}\right) \notin \mathcal{C E}$ because $u_{1}=\frac{11}{3}$ implies $p_{1}=\frac{37}{3}$ and $u_{2}=5$ implies $p_{1}=19 / 3$. To see that $\left(\frac{11}{3}, 5, \frac{28}{3}\right) \in \mathcal{S W}$ consider $\Gamma=\left(\begin{array}{ll}\Gamma_{111} & \Gamma_{121} \\ \Gamma_{211} & \Gamma_{221}\end{array}\right)=\left(\begin{array}{cc}\frac{19}{3} & 2 \\ \frac{19}{3} & 2\end{array}\right), \mathcal{C}=\left\{b_{1}, s_{1}\right\}$ (it is immediate to see that the other coalitions can not $S W$-block $\left.\left(\frac{11}{3}, 5, \frac{28}{3}\right)\right)$, and $\widehat{A}=\left(\begin{array}{ll}2 & 0 \\ 2 & 0\end{array}\right)$. Observe that $\widehat{A}$ is $S W$-compatible with $\mathcal{C}$. Then,

$$
13>\tau_{111} \widehat{A}_{111}+\left(\Gamma_{211}-r_{11}\right) \widehat{A}_{211}=4 \cdot 2+\left(\frac{19}{3}-4\right) \cdot 2=\frac{38}{3}
$$

and for any other different assignment $A^{\prime}$ that is $S W$-compatible with $\mathcal{C}$,

$$
13>\tau_{111} A_{111}^{\prime}+\left(\Gamma_{211}-r_{11}\right) A_{211}^{\prime}
$$

Hence, $\left(\frac{11}{3}, 5, \frac{28}{3}\right) \in \mathcal{S W} \backslash \mathcal{C E}$.

Competitive equilibrium presupposes that agents are price-takers. This assumption makes sense only when the number of agents is large and individual quantity decisions are insignificant. Thus, and at the light of $\varnothing \neq \mathcal{C} \mathcal{E} \subsetneq \mathcal{S W}$, it is natural to ask whether the set of Set-wise stable payoffs and the set of competitive equilibrium payoffs are approximately the same when the number of agents becomes large. To enlarge the market, we follow a procedure with a long tradition in Economics which consists of replicating the market. ${ }^{6}$ Given a market $M=(\mathcal{B}, \mathcal{G}, \mathcal{S}, V, d, R, Q)$ and a strictly positive integer $\rho$ we will consider the $\rho$-fold replicated market $\rho M$ to be composed of $\rho$ agents of each type. For two buyers

\footnotetext{
${ }^{6}$ It started by Edgeworth (1881) and pursued by Debreu and Scarf (1963) for classical economies with production and by Owen (1975) for linear production games, among others. A linear production game consists of a set of players, each with an endowment (non necessarily integer valued) of $m$ goods that can only be used to produce in a linear way units of $p$ different goods for which there are competitive markets. Owen (1975) shows that the sequence of Cores of replicated linear production games converges to the set of competitive equilibrium payoffs. Moreover, Owen (1975) also shows that if the competitive equilibrium price is unique then the Core of a large but finitely replicated game coincides with the (unique) competitive equilibrium payoff. See also Kaneko and Wooders (1982) and Wooders (1983, 1994 and 2010) for Core convergence results for games (with and without side payments) and markets.
} 
$i_{\alpha} \in \mathcal{B}_{\alpha}$ and $i_{\alpha^{\prime}} \in \mathcal{B}_{\alpha^{\prime}}$ (in replicas $\alpha$ and $\alpha^{\prime}$, respectively) to be of the same type we require them to have the same valuations of all goods (i.e., $v_{i_{\alpha} j}=v_{i_{\alpha^{\prime}} j}=v_{i j}$ for all $j \in \mathcal{G}$ ) and the same maximal demands (i.e., $d_{i_{\alpha}}=d_{i_{\alpha^{\prime}}}=d_{i}$ ). For two sellers $k_{\alpha} \in \mathcal{S}_{\alpha}$ and $k_{\alpha^{\prime}} \in \mathcal{S}_{\alpha^{\prime}}$ (in replicas $\alpha$ and $\alpha^{\prime}$, respectively) to be of the same type we require them to have the same reservation prices of all goods (i.e., $r_{j k_{\alpha}}=r_{j k_{\alpha^{\prime}}}=r_{j k}$ for all $j \in \mathcal{G}$ ) and the same amounts of all goods (i.e., $q_{j k_{\alpha}}=q_{j k_{\alpha^{\prime}}}=q_{j k}$ for all $j \in \mathcal{G}$ ).

Our main result of the paper states that the Set-wise stable set of the 2 -fold replicated market has the equal treatment property (all replicated agents receive the same payoff) and coincides with the set of competitive equilibrium payoffs.

Theorem 1 Let $(u, w),(\bar{u}, \bar{w}) \in \mathbb{R}_{+}^{\mathcal{B}} \times \mathbb{R}_{+}^{\mathcal{S}}$ be two payoff vectors of market $M=(\mathcal{B}, \mathcal{G}, \mathcal{S}, V, d, R, Q)$. Then,

$$
((u, w),(\bar{u}, \bar{w})) \in \mathcal{S} \mathcal{W}^{2 M} \text { if and only if }(u, w)=(\bar{u}, \bar{w}) \in \mathcal{C} \mathcal{E} .
$$

Proof See the Appendix.

The idea of the proof is as follows. Fix a market $M=(\mathcal{B}, \mathcal{G}, \mathcal{S}, V, d, R, Q)$. First, by Lemma 2 in the Appendix, we show that for any replica a Set-wise stable payoff has the equal treatment property: all agents of the same type receive the same payoff (this is a classical property that also holds for the Core of replicated markets). Second, by definition, a Set-wise stable payoff $(u, w)$ can be obtained by a set of trade agreements that are $S W$-unblocked; that is, there exists a distribution matrix $\Gamma$ such that no coalition $\mathcal{C}$ can $S W$-block $(u, w)$ because

$$
\sum_{i \in \mathcal{B}^{\mathcal{C}}} u_{i}+\sum_{k \in \mathcal{S}^{\mathcal{C}}} w_{k} \geq \phi^{M}(\mathcal{C}, \widehat{A}, \Gamma)
$$

where $\widehat{A}$ is $S W$-compatible with $\mathcal{C}$. Third, and as a consequence of Lemma 4 in the Appendix, we show that one of the unblocked set of trade agreements (described by a distribution matrix $\Gamma$ ) is anonymous because there exists $p=\left(p_{j}\right)_{j \in \mathcal{G}}$ such that for all $j \in \mathcal{G}, p_{j}=\Gamma_{i j k}$ for all $(i, k) \in \mathcal{B} \times \mathcal{S}$ and $\Gamma$ can be replaced by $p$ in condition (12); namely,

$$
\sum_{i \in \mathcal{B}^{\mathcal{C}}} u_{i}+\sum_{k \in \mathcal{S}^{\mathcal{C}}} w_{k} \geq \phi^{M}(\mathcal{C}, \widehat{A}, p)
$$

Fourth, for any payoff vector $(u, w)$ in market $M$ with the property that the payoff vector $((u, w),(u, w))$ is a Set-wise stable payoff of the two replica market $2 M$, the vector $p$ that satisfies condition (13) is a competitive equilibrium price of market $M$. Finally, in Lemma 6 in the Appendix, we show that if a payoff vector $(u, w)$ satisfies condition (13) for a 
competitive equilibrium price vector $p$ then $(u, w)$ is a competitive equilibrium payoff of market $M$.

We use Example 1 again to illustrate how a payoff $(u, w) \in \mathcal{S} \mathcal{W} \backslash \mathcal{C E}$ can be already $S W$-blocked in the two replicated market.

Example 1 (continued) We have already showed that $\left(u_{1}, u_{2}, w_{1}\right)=\left(\frac{11}{3}, 5, \frac{28}{3}\right) \in \mathcal{S} \mathcal{W} \backslash \mathcal{C E}$. Hence, by Theorem $1,\left(\left(\frac{11}{3}, 5, \frac{28}{3}\right),\left(\frac{11}{3}, 5, \frac{28}{3}\right)\right) \notin \mathcal{S} \mathcal{W}^{2 M}$. To illustrate how it is $S W$-blocked, consider any distribution matrix such that $\Gamma_{2_{1} 11_{1}}=7.5$, the coalition $\mathcal{C}=\left\{b_{1_{1}}, b_{1_{2}}, s_{1_{1}}\right\}$, and the assignment

$$
\widehat{A}=\left(\begin{array}{cccc}
\widehat{A}_{1_{1} 11_{1}} & \widehat{A}_{1_{1} 21_{1}} & \widehat{A}_{1_{1} 11_{2}} & \widehat{A}_{1_{1} 21_{2}} \\
\widehat{A}_{2_{111_{1}}} & \widehat{A}_{2_{121}} & \widehat{A}_{2_{1} 21_{2}} & \widehat{A}_{2_{1} 21_{2}} \\
\widehat{A}_{1_{2} 11_{1}} & \widehat{A}_{1_{2} 11_{1}} & \widehat{A}_{1_{2} 11_{2}} & \widehat{A}_{1_{2} 11_{2}} \\
\widehat{A}_{2_{2} 11_{1}} & \widehat{A}_{2_{2} 11_{1}} & \widehat{A}_{2_{2} 11_{2}} & \widehat{A}_{2_{2} 11_{2}}
\end{array}\right)=\left(\begin{array}{cccc}
1 & 1 & 0 & 0 \\
1 & 0 & 0 & 0 \\
2 & 0 & 0 & 0 \\
0 & 0 & 3 & 0
\end{array}\right)
$$

$S W$-compatible with $\mathcal{C}$. Then,

$$
\begin{aligned}
\phi^{2 M}(\mathcal{C}, \widehat{A}, \Gamma) & =\tau_{1_{1} 11_{1}} \cdot \widehat{A}_{1_{1} 11_{1}}+\tau_{1_{1} 21_{1}} \cdot \widehat{A}_{1_{1} 21_{1}}+\tau_{1_{2} 11_{1}} \cdot \widehat{A}_{1_{2} 11_{1}}+\left(\Gamma_{2_{1} 11_{1}}-r_{11_{1}}\right) \cdot \widehat{A}_{2_{1} 11_{1}} \\
& =4 \cdot 1+2 \cdot 1+4 \cdot 2+(7.5-4) \cdot 1 \\
& =17.5 \\
& >\frac{50}{3}=u_{1_{1}}+u_{1_{2}}+w_{1_{1}} .
\end{aligned}
$$

Hence, $\mathcal{C} S W$-blocks $\left(\left(\frac{11}{3}, 5, \frac{28}{3}\right),\left(\frac{11}{3}, 5, \frac{28}{3}\right)\right)$. Observe that the expression $\left(\Gamma_{2_{1} 11_{1}}-r_{11_{1}}\right)$. $\widehat{A}_{21_{11}}=(7.5-4) \cdot 1$ shows that one member of the blocking coalition (seller $\left.1_{1}\right)$ keeps selling 1 unit of good 1 to buyer $2_{1}$, who is not a member of the blocking coalition $\mathcal{C}$.

\subsection{Core}

Let $M=(\mathcal{B}, \mathcal{G}, \mathcal{S}, V, d, R, Q)$ be a market and let $\mathcal{C} \subseteq \mathcal{B} \cup \mathcal{S}$ be a coalition. Remember that we denote the subsets of buyers and sellers in $\mathcal{C}$ by $\mathcal{B}^{\mathcal{C}}=\mathcal{C} \cap \mathcal{B}$ and $\mathcal{S}^{\mathcal{C}}=\mathcal{C} \cap \mathcal{S}$, respectively. The submarket $M^{\mathcal{C}}$ is the (natural) restriction of market $M$ to coalition $\mathcal{C}$; namely, $M^{\mathcal{C}}$ is the market $\left(\mathcal{B}^{\mathcal{C}}, \mathcal{G}^{\mathcal{C}}, \mathcal{S}^{\mathcal{C}}, V^{\mathcal{C}}, d^{\mathcal{C}}, R^{\mathcal{C}}, Q^{\mathcal{C}}\right)$, where $\mathcal{G}^{\mathcal{C}}=\{j \in \mathcal{G}$ |there exists $k \in \mathcal{S}^{\mathcal{C}}$ such that $\left.q_{j k}>0\right\}, V^{\mathcal{C}}=\left(v_{i j}\right)_{(i j) \in \mathcal{B}^{\mathcal{C}} \times \mathcal{G}^{\mathcal{C}}}, d^{\mathcal{C}}=\left(d_{i}\right)_{i \in \mathcal{B}^{\mathcal{C}}}, R^{\mathcal{C}}=\left(r_{j k}\right)_{(j, k) \in \mathcal{G}^{\mathcal{C}} \times \mathcal{S}^{\mathcal{C}}}$, and

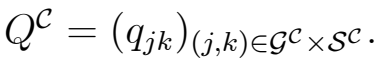

Definition 6 A feasible assignment $A$ is Core-compatible with coalition $\mathcal{C}$ if $A_{i j k} \neq 0$ implies $\{i, k\} \subset \mathcal{C}$. 
That is, a feasible assignment $A$ is Core-compatible with $\mathcal{C}$ if all members of $\mathcal{C}$ interact only among themselves. Let $A$ be an assignment Core-compatible with coalition $\mathcal{C}$ and denote by $A^{\mathcal{C}}$ the feasible assignment for submarket $M^{\mathcal{C}}$, where $A^{\mathcal{C}}=\left(A_{i j k}\right)_{(i, j, k) \in \mathcal{B}^{\mathcal{C}} \times \mathcal{G}^{\mathcal{C}} \times \mathcal{S}^{\mathcal{C}}}$. When the reference coalition is clear from the context we often omit the superscript $\mathcal{C}$. Denote by $\mathcal{F}^{\mathcal{C}}$ the set of optimal assignments of market $M^{\mathcal{C}}$; i.e.,

$$
\mathcal{F}^{\mathcal{C}}=\left\{A^{\mathcal{C}} \in \mathbb{Z}_{+}^{B \times G \times S} \mid T^{M^{\mathcal{C}}}\left(A^{\mathcal{C}}\right) \geq T^{M^{\mathcal{C}}}\left(A^{\mathcal{C}}\right) \text { for any feasible assignment } A^{\mathcal{C}}\right\} .
$$

Fix a market $M=(\mathcal{B}, \mathcal{G}, \mathcal{S}, V, d, R, Q)$. To define a cooperative game $v$ with transferable utility associated to $M$, let $\mathcal{C} \subseteq \mathcal{B} \cup \mathcal{S}$ be a coalition and set

$$
v(\mathcal{C})=T^{M^{\mathcal{C}}}\left(A^{\mathcal{C}}\right)
$$

where $A^{\mathcal{C}}$ is any optimal assignment of submarket $M^{\mathcal{C}}$. Namely, $v(\mathcal{C})$ is the maximal total utility that members of $\mathcal{C}$ can guarantee by exchanging their resources only among themselves. Obviously, $v(\mathcal{C})=0$ for all $\mathcal{C}$ such that either $\mathcal{B}^{\mathcal{C}}=\varnothing$ or $\mathcal{S}^{\mathcal{C}}=\varnothing$, and hence, $v(\varnothing)=0$. Moreover, $v(\{i\})=0$ for all $i \in \mathcal{B}$ and $v(\{k\})=0$ for all $k \in \mathcal{S}$.

A pair $(u, w) \in \mathbb{R}^{B} \times \mathbb{R}^{S}$ is a (feasible) payoff of market $M=(\mathcal{B}, \mathcal{G}, \mathcal{S}, V, d, R, Q)$ if

$$
\sum_{i \in \mathcal{B}} u_{i}+\sum_{k \in \mathcal{S}} w_{k}=v(\mathcal{B} \cup \mathcal{S})
$$

A payoff of market $M$ is a distribution among agents of the total gains from trade at any optimal assignment of market $M$.

Definition 7 A payoff $(u, w) \in \mathbb{R}^{B} \times \mathbb{R}^{S}$ of market $M=(\mathcal{B}, \mathcal{G}, \mathcal{S}, V, d, R, Q)$ is Core-blocked by coalition $\mathcal{C} \subseteq \mathcal{B} \cup \mathcal{S}$ if

$$
\sum_{i \in \mathcal{B}^{\mathcal{C}}} u_{i}+\sum_{k \in \mathcal{S}^{\mathcal{C}}} w_{k}<v(\mathcal{C})
$$

Definition 8 A payoff $(u, w) \in \mathbb{R}^{B} \times \mathbb{R}^{S}$ of market $M=(\mathcal{B}, \mathcal{G}, \mathcal{S}, V, d, R, Q)$ belongs to the Core if there does not exist a coalition $\mathcal{C} \subset \mathcal{B} \cup \mathcal{S}$ such that $(u, w)$ is Core-blocked by $\mathcal{C}$.

Let $\mathcal{C} o$ be the set of payoffs belonging to the Core of market $M=(\mathcal{B}, \mathcal{G}, \mathcal{S}, V, d, R, Q)$. When we want to emphasize market $M$ we write $\mathcal{C} O^{M}$. To establish the relationship between the Core and the set of Set-wise stable payoffs, let $(u, w) \in \mathbb{R}^{B} \times \mathbb{R}^{S}$ be a payoff of market $M$ and assume that coalition $\mathcal{C}$ Core-blocks $(u, w)$. Let $A^{\mathcal{C}} \in \mathcal{F}^{\mathcal{C}}$ be arbitrary. Then,

$$
\sum_{i \in \mathcal{B}^{\mathcal{C}}} u_{i}+\sum_{k \in \mathcal{S}^{\mathcal{C}}} w_{k}<v(\mathcal{C})=T^{M^{\mathcal{C}}}\left(A^{\mathcal{C}}\right)
$$


Let $\widehat{A}$ be the feasible assignment where, for all $(i, j, k) \in \mathcal{B} \times \mathcal{G} \times \mathcal{S}$,

$$
\widehat{A}_{i j k}= \begin{cases}A_{i j k}^{\mathcal{C}} & \text { if }(i, k) \in \mathcal{B}^{\mathcal{C}} \times \mathcal{S}^{\mathcal{C}} \\ 0 & \text { otherwise. }\end{cases}
$$

Then, $\widehat{A}$ is a feasible assignment $S W$-compatible with $\mathcal{C}$ and for any distribution matrix $\Gamma$,

$$
\sum_{i \in \mathcal{B}^{\mathcal{C}}} u_{i}+\sum_{k \in \mathcal{S}^{\mathcal{C}}} w_{k}<\phi^{M}(\mathcal{C}, \widehat{A}, \Gamma)=T^{M^{\mathcal{C}}}\left(A^{\mathcal{C}}\right) .
$$

Hence, coalition $\mathcal{C}$ SW-blocks $(u, w)$. Thus, the Set-wise stable set is a subset of the Core. For further reference, we state this fact below as Remark 3.

Remark 3 For any market the Set-wise stable set is a subset of the Core.

Thus, we have already showed that the statement of the following corollary holds. ${ }^{7}$

Corollary 1 For every market $M, \varnothing \neq \mathcal{C} \mathcal{E} \subset \mathcal{S W} \subset \mathcal{C}$ o. Moreover, the two inclusions may be strict.

In Example 1 above we have already showed that the first inclusion may be strict. To show that the second inclusion may also be strict we return again to Example 1.

Example 1 (continued) Consider the vector $\left(u_{1}, u_{2}, w_{1}\right)=(4.5,10,3.5)$. To see that it belongs to the Core of $M$ it is enough to check that for any coalition that can obtain strictly positive gains from trade can not Core-blocked it. Indeed,

$$
\begin{aligned}
& v\left(\left\{b_{1}, b_{2}, s_{1}\right\}\right)=18=4.5+10+3.5=u_{1}+u_{2}+w_{1} . \\
& v\left(\left\{b_{1}, s_{1}\right\}\right)=8=4.5+3.5=u_{1}+w_{1} . \\
& v\left(\left\{b_{2}, s_{1}\right\}\right)=12<10+3.5=13.5=u_{2}+w_{1} .
\end{aligned}
$$

Hence, $(4.5,10,3.5) \in \mathcal{C}$ o. Now, to see that $(4.5,10,3.5) \notin \mathcal{S W}$ consider any distribution matrix $\Gamma=\left(\begin{array}{ll}\Gamma_{111} & \Gamma_{121} \\ \Gamma_{211} & \Gamma_{221}\end{array}\right)$. By definition, $8=v_{21} \geq \Gamma_{211} \geq r_{11}=4$. Assume first that $\Gamma_{211}=4$. Then, the optimal assignment $A=\left(\begin{array}{ll}1 & 1 \\ 3 & 0\end{array}\right)$ is itself $S W$-compatible with $\mathcal{C}=\left\{b_{2}\right\}$ and $u_{2}=10<\left(v_{21}-\Gamma_{211}\right) \cdot A_{211}+\left(v_{22}-\Gamma_{221}\right) \cdot A_{221}=4 \cdot 3+\left(v_{22}-\Gamma_{221}\right) \cdot 0=12$. Hence, $\mathcal{C}=\left\{b_{2}\right\} S W$-blocks $(4.5,10,3.5)$. Assume now that $\Gamma_{211}>4$. Then, consider the assignment $\widehat{A}=\left(\begin{array}{ll}2 & 0 \\ 2 & 0\end{array}\right)$ which is $S W$-compatible with $\mathcal{C}=\left\{b_{1}, s_{1}\right\}$ and note that

\footnotetext{
${ }^{7}$ The same inclusion relationships hold in the time-sharing assignment games considered by Sotomayor (2009 and 2011).
} 
$u_{1}+w_{1}=8<\tau_{111} \cdot \widehat{A}_{111}+\left(\Gamma_{211}-r_{11}\right) \cdot \widehat{A}_{211}=4 \cdot 2+\left(\Gamma_{211}-4\right) \cdot 2$, where the inequality follows from $\Gamma_{211}>4$. Hence, $\mathcal{C}=\left\{b_{1}, s_{1}\right\} S W$-blocks $(4.5,10,3.5)$. Thus $(4.5,10,3.5) \notin \mathcal{S W}$.

We want to emphasize again, at the light of the example, that the notions of Set-wise stability is more natural than Core. When seller 1 , as member of the coalition $\left\{b_{1}, s_{1}\right\}$ Core-blocks $(4.5,10,3.5)$ has to stop selling 3 units of good one to $b_{2}$, while when $\left\{b_{1}, s_{1}\right\}$ $S W$-blocks $(4.5,10,3.5), s_{1}$ can keep selling 2 units of good one to $b_{2}$.

Our second limit result states that, for every market $M=(\mathcal{B}, \mathcal{G}, \mathcal{S}, V, d, R, Q)$, the sequence of Cores of the $\rho M$ markets converges, when $\rho \rightarrow \infty$, to the set of competitive equilibrium payoffs of the replicated market. To state it we need the following lemma saying that the classical result stating that any payoff vector in the Core of a replicated market assigns the same payoff to all agents of the same type also holds in this setting. ${ }^{8}$

Lemma 1 Let $M=(\mathcal{B}, \mathcal{G}, \mathcal{S}, V, d, R, Q)$ be a market and let $\rho \geq 2$. Then,

$$
\mathcal{C} o^{\rho M} \subset\left\{\left(u^{\rho}, w^{\rho}\right) \equiv(\underbrace{(u, w), \ldots,(u, w)}_{\rho \text {-times }}) \in\left(\mathbb{R}^{B_{1}} \times \mathbb{R}^{S_{1}}\right) \times \ldots \times\left(\mathbb{R}^{B_{\rho}} \times \mathbb{R}^{S_{\rho}}\right) \mid(u, w) \in \mathcal{C} o^{M}\right\} .
$$

Proof See the Appendix.

With some abuse of the language we will say that a payoff vector $(u, w) \in \mathbb{R}^{B} \times \mathbb{R}^{S}$ is in the Core of the $\rho$-replicated market if $\left(u^{\rho}, w^{\rho}\right) \in \mathcal{C} o^{\rho M}$.

Theorem 2 Let $(u, w) \in \mathbb{R}^{B} \times \mathbb{R}^{S}$ be a payoff of market $M=(\mathcal{B}, \mathcal{G}, \mathcal{S}, V, d, R, Q)$. Then, $(u, w)$ is in the Core of the $\rho$-fold replicated market for all $\rho \geq 1$ if and only if $(u, w)$ is a competitive equilibrium payoff of market $M$.

Lemma 1 says that for any replica $\rho$ the payoff vectors in the Core of market $\rho M$ have the equal treatment property. Theorem 2 says that the payoff vector that belongs to the Core of all replicated markets are those obtained by replicating competitive equilibrium payoffs of the original market. With an abuse of notation, Theorem 2 says that for any market $M, \bigcap_{\rho=1}^{\infty} \mathcal{C} o^{\rho M}=\mathcal{C E}^{M}$. Moreover, the convergence is monotonically decreasing: if $\left(u^{\rho}, w^{\rho}\right) \in \mathcal{C} o^{\rho M}$ then $\left(u^{\rho+1}, w^{\rho+1}\right) \in \mathcal{C}_{O}{ }^{(\rho+1) M}$, since any coalition blocking $\left(u^{\rho}, w^{\rho}\right)$ should also block $\left(u^{\rho+1}, w^{\rho+1}\right)$.

Theorem 2 can be proved by adapting Owen (1975)'s proof of the convergence of the Core to the set of competitive equilibrium payoffs for linear programming games, and therefore

\footnotetext{
${ }^{8}$ See Debreu and Scarf (1963) and Owen (1975) for this equal treatment result in classical economies with production and in linear production games, respectively.
} 
we omit it. ${ }^{9}$

To illustrate the two different blocking notions we return to Example 1.

Example 1 (continued) We have already showed that $\left(u_{1}, u_{2}, w_{1}\right)=\left(\frac{11}{3}, 5, \frac{28}{3}\right) \in \mathcal{S} \mathcal{W} \backslash \mathcal{C E}$. Hence, by Theorem $1,\left(\left(\frac{11}{3}, 5, \frac{28}{3}\right),\left(\frac{11}{3}, 5, \frac{28}{3}\right)\right) \notin \mathcal{S} \mathcal{W}^{2 M}$. We have already illustrated that coalition $\mathcal{C}=\left\{b_{1_{1}}, b_{1_{2}}, s_{1_{1}}\right\} S W$-blocks it for a particular distribution matrix $\Gamma$. To illustrate now the notion of Core in a two-fold replicated market, consider again the coalition $\mathcal{C}=\left\{b_{1_{1}}, b_{1_{2}}, s_{1_{1}}\right\}$ that $S W$-blocks $\left(\left(\frac{11}{3}, 5, \frac{28}{3}\right),\left(\frac{11}{3}, 5, \frac{28}{3}\right)\right) \notin \mathcal{S} \mathcal{W}^{2 M}$, and whose value is $v(\mathcal{C})=16$. Since, $u_{1_{1}}+u_{1_{2}}+w_{1_{1}}=\frac{50}{3}, \mathcal{C}$ does not Core-blocks $\left(\left(\frac{11}{3}, 5, \frac{28}{3}\right),\left(\frac{11}{3}, 5, \frac{28}{3}\right)\right)$. By Theorem 1 , and since $\left(\frac{11}{3}, 5, \frac{28}{3}\right) \notin \mathcal{C} \mathcal{E}$ we already know that for any distribution matrix one can find a coalition and a $S W$-compatible assignment that $S W$-blocks $\left(\left(\frac{11}{3}, 5, \frac{28}{3}\right),\left(\frac{11}{3}, 5, \frac{28}{3}\right)\right)$. The fact that this is not an easy task illustrates the power of Theorem 1 because to show that $\left(\frac{11}{3}, 5, \frac{28}{3}\right) \notin \mathcal{C} \mathcal{E}$ was very easy.

Theorem 3 shows that a result similar to Theorem 1 does not hold for the Core. For each number $\rho$ of replicas there exists a market $M=(\mathcal{B}, \mathcal{G}, \mathcal{S}, V, d, R, Q)$ for which the Core of the $\rho$-fold replicated market contains a payoff that is not a competitive equilibrium payoff.

Theorem 3 Let $\rho \in \mathbb{Z}_{+} \backslash\{0\}$. Then, there exist a market $M=(\mathcal{B}, \mathcal{G}, \mathcal{S}, V, d, R, Q)$ and a payoff vector $(u, w) \notin \mathcal{C E}$ such that $\left(u^{\rho}, w^{\rho}\right) \in \mathcal{C} o^{\rho M}$.

Proof See the Appendix.

\section{Concluding Remarks}

The convergence of the Core to competitive equilibria is a pervasive phenomenon that holds in many settings. Our Theorem 2 is a new result in this line. The convergence in most of these results is only asymptotic. Owen (1975) is one of the few exceptions where the convergence is finite. Our main contribution, Theorem 1, is that for generalized assignment games the convergence of Set-wise stable payoffs to competitive equilibria only requires two replica. Since Set-wise stability is a more appropriated notion in this context, our result suggests that all outcomes that are robust to coalitional deviations in the sense of Set-wise stability can be obtained as competitive equilibrium outcomes, even in markets with an small number of agents. Therefore, in relatively small markets with indivisibilities

\footnotetext{
${ }^{9}$ The interested reader can find a detailed proof of Theorem 2 in the authors' websites.
} 
similar to generalized assignment games (for instance, markets for intermediate goods), competitive outcomes are those that are immune to secession by subsets of agents, since they will coincide with the of Set-wise stable outcomes. We think that this result may be important for future research studying markets (or general allocation problems) in which agents may simultaneously be interacting with other agents in different ways and hence, the convergence of the Set-wise stable set would be the relevant one to evaluate how robust is the principle that competitive equilibrium outcomes coincide with the set of outcomes that are immune to coalitional deviations.

We leave for future research two open problems. First, the analysis of agents' strategic behavior in small markets when agents recognize their market power and consequently they may not take prices as given. Second, the analysis of an intermediate cooperative notion, ${ }^{10}$ in which a member of a blocking coalition can either keep all agreements with a non-member or break all of them; this set would be a subset of the Core and contain the Set-wise stable set, but we do not know whether its convergence to competitive equilibria would be like the Core (infinite) or like Set-wise stability (finite).

\section{Acknowledgements}

We are grateful to an Associate Editor and three referees of this journal for many comments and suggestions. Massó acknowledges financial support from the Spanish Ministry of Economy and Competitiveness, through the Severo Ochoa Programme for Centres of Excellence in R\&D (SEV-2011-0075) and FEDER grant ECO2008-04756 (Grupo Consolidado-C), and from the Generalitat de Catalunya, through the prize "ICREA Acadèmia" for excellence in research and grant SGR2009-419. He also acknowledges the support of MOVE, where he is an affiliated researcher. The work of Neme is partially supported by the Universidad Nacional de San Luis grant 319502 and by the CONICET grant PIP 112-200801-00655.

\footnotetext{
${ }^{10}$ Sotomayor (2011) names it "with rigid agreements" and studies it for a time-sharing assignment game.
} 


\section{References}

[1] Camiña, E., 2006. A generalized assignment game. Math Soc Sci 52, 152-161.

[2] Dantzig, G., 1963. Linear Programming and Extensions. Princeton University Press, Princeton.

[3] Debreu, G., Scarf, H., 1963. A limit theorem on the Core of an economy. Int Econ Rev 4, 235-246.

[4] Edgeworth, F.Y., 1881. Mathematical Psychics. London: Kegan Paul.

[5] Jaume, D., Massó, J., Neme, A., 2012. The multi-partners assignment game with heterogeneous sales and multi-unit demands: competitive equilibria. Math Meth Oper Res 76, 161-187.

[6] Kaneko, M., Wooders, M., 1982. Cores of partitioning games. Math Soc Sci 3, 313-327.

[7] Klaus, B., Walzl, M., 2009. Stable many-to-many matchings with contracts. J Math Econ 45, 422-434.

[8] Milgrom, P., 2009. Assignment messages and exchanges. Am Econ Rev: Micro 1, 95113.

[9] Owen, G., 1975. On the core of linear production games. Math Program 9, 358-370.

[10] Shapley, L. S., Shubik, M., 1972. The assignment game I: the core. Int J Game Theory $1,111-130$.

[11] Sotomayor, M., 1992. The multiple partners game, in Majumdar, M. (Ed.) Equilibrium and Dynamics: Essays in Honor to David Gale. Macmillian, pp. 322-336.

[12] Sotomayor, M., 1999a. The lattice structure of the set of stable outcomes of the multiple partners assignment game. Int J Game Theory 28, 567-583.

[13] Sotomayor, M., 1999b. Three remarks on the many-to-many stable matching problem. Math Soc Sci 38, 55-70.

[14] Sotomayor, M., 2007. Connecting the cooperative and competitive structures of the multiple-partners assignment game. J Eco Theory 134, 155-174. 
[15] Sotomayor, M., 2009. Correlating new cooperative and competitive concepts in the time-sharing assignment game. Mimeo.

[16] Sotomayor, M., 2011. Correlating the competitive and cooperative structures of the time-sharing assignment game under rigid agreements. Mimeo.

[17] Wooders, M., 1983. The epsilon core of a large replica game. J Math Econ 11, 277-300.

[18] Wooders, M., 1994. Equivalence of games and markets. Econometrica 62, 1141-1160.

[19] Wooders, M., 2010. Cores of many-player games; nonemptiness and equal treatment. Rev Econ Des 14, 131-162. 


\section{Appendix: Preliminaries and Omitted Proofs}

We start with some preliminaries. Let $M=(\mathcal{B}, \mathcal{G}, \mathcal{S}, V, d, R, Q)$ be a market and $\mathcal{C}$ be a coalition. Consider the primal linear problem to which any optimal assignment $A^{\mathcal{C}} \in \mathcal{F}^{\mathcal{C}}$ is a solution.

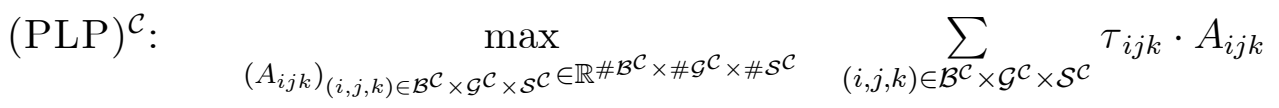

$$
\begin{aligned}
& \text { s. t. (P.1) } \sum_{(j, k) \in \mathcal{G}^{\mathcal{C}} \times \mathcal{S}^{\mathcal{C}}} A_{i j k} \leq d_{i} \quad \text { for all } i \in \mathcal{B}^{\mathcal{C}} \text {, } \\
& \text { (P.2) } \quad \sum_{i \in \mathcal{B}^{\mathcal{C}}} A_{i j k} \leq q_{j k} \quad \text { for all }(j, k) \in \mathcal{G}^{\mathcal{C}} \times \mathcal{S}^{\mathcal{C}} \text {, } \\
& \text { (P.3) } \quad A_{i j k} \geq 0 \quad \text { for all }(i, j, k) \in \mathcal{B}^{\mathcal{C}} \times \mathcal{G}^{\mathcal{C}} \times \mathcal{S}^{\mathcal{C}} \text {. }
\end{aligned}
$$

The dual linear problem associated to $(\mathrm{PLP})^{\mathcal{C}}$ is the following.

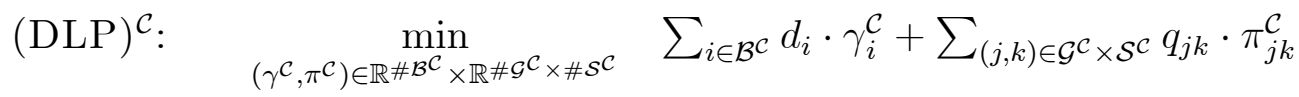

$$
\begin{aligned}
& \text { s. t. (D.1) } \gamma_{i}^{\mathcal{C}}+\pi_{j k}^{\mathcal{C}} \geq \tau_{i j k} \quad \text { for all }(i, j, k) \in \mathcal{B}^{\mathcal{C}} \times \mathcal{G}^{\mathcal{C}} \times \mathcal{S}^{\mathcal{C}} \text {, } \\
& \text { (D.2) } \quad \gamma_{i}^{\mathcal{C}} \geq 0 \quad \text { for all } i \in \mathcal{B}^{\mathcal{C}} \text {, } \\
& \text { (D.3) } \quad \pi_{j k}^{\mathcal{C}} \geq 0 \quad \text { for all }(j, k) \in \mathcal{G}^{\mathcal{C}} \times \mathcal{S}^{\mathcal{C}} \text {. }
\end{aligned}
$$

Let $D^{\mathcal{C}}$ be the set of all solutions of the $(\mathrm{DLP})^{\mathcal{C}}$. It is well-known that $D^{\mathcal{C}}$ is non-empty. We will denote the set $D^{\mathcal{B} \cup \mathcal{S}}$ by $D$ and $\left(\gamma^{\mathcal{B} \cup \mathcal{S}}, \pi^{\mathcal{B} \cup \mathcal{S}}\right)$ by $(\gamma, \pi) \in D$. Then, it is immediate to check that the following implication holds.

$$
\text { If }(\gamma, \pi) \in D \text { then }\left(\left(\gamma_{i}\right)_{i \in \mathcal{B}^{\mathcal{C}}},\left(\pi_{j k}\right)_{(j, k) \in \mathcal{G}^{\mathcal{C}} \times \mathcal{S}^{\mathcal{C}}}\right) \in D^{\mathcal{C}} \text {. }
$$

Let $M=(\mathcal{B}, \mathcal{G}, \mathcal{S}, V, d, R, Q)$ be a market and $(\gamma, \pi)$ be a vector satisfying (D.1), (D.2), and (D.3) for $\mathcal{C}=\mathcal{B} \cup \mathcal{S}$. We write $T D^{M}(\gamma, \pi)$ to denote the value of the objective function of the $(\mathrm{DLP})^{\mathcal{B} \cup \mathcal{S}}$ at $(\gamma, \pi)$; that is,

$$
T D^{M}(\gamma, \pi)=\sum_{i} d_{i} \cdot \gamma_{i}+\sum_{j k} q_{j k} \cdot \pi_{j k}
$$


The Strong Duality Theorem (SDT) of Linear Programming applied to our setting says the following (see Dantzig, 1963).

Strong Duality Theorem Let $M=(\mathcal{B}, \mathcal{G}, \mathcal{S}, V, d, R, Q)$ be a market and assume $A$ is a feasible assignment and $(\gamma, \pi)$ satisfies (D.1), (D.2), and (D.3) for $\mathcal{C}=\mathcal{B} \cup \mathcal{S}$. Then,

$$
A \in \mathcal{F} \text { and }(\gamma, \pi) \in D \text { if and only if } T^{M}(A)=T D^{M}(\gamma, \pi) .
$$

Proposition 1 Let $p \in \mathcal{P}$ be a competitive equilibrium price vector of market $M=$ $(\mathcal{B}, \mathcal{G}, \mathcal{S}, V, d, R, Q)$. Then, $(u(p), w(p)) \in \mathcal{S W}$.

Proof Let $p \in \mathcal{P}$ and assume $(u(p), w(p)) \notin \mathcal{S} \mathcal{W}$. Then, for every distribution matrix $\Gamma=\left(\Gamma_{i j k}\right)_{(i, j, k) \in \mathcal{B} \times \mathcal{G} \times \mathcal{S}}$ there exists a coalition $\mathcal{C} \subset \mathcal{B} \cup \mathcal{S}$ that $S W$-blocks it. Hence, there exists a feasible assignment $\widehat{A}$ that is $S W$-compatible with $\mathcal{C}$ such that

$$
\sum_{i \in \mathcal{B}^{\mathcal{C}}} u_{i}(p)+\sum_{k \in \mathcal{S}^{\mathcal{C}}} w_{k}(p)<\phi^{M}(\mathcal{C}, \widehat{A}, \Gamma)
$$

In particular, consider the distribution matrix $\Gamma=\left(\Gamma_{i j k}\right)_{(i, j, k) \in \mathcal{B} \times \mathcal{G} \times \mathcal{S}}$ where for each $(i, j, k) \in$ $\mathcal{B} \times \mathcal{G} \times \mathcal{S}, \Gamma_{i j k}=p_{j}$. Then, there must exist a feasible assignment $\widehat{A}$ that is $S W$-compatible with $\mathcal{C}$ such that ${ }^{11}$

$$
\sum_{i \in \mathcal{B}^{\mathcal{C}}} u_{i}(p)+\sum_{k \in \mathcal{S}^{\mathcal{C}}} w_{k}(p)<\phi^{M}(\mathcal{C}, \widehat{A}, p) .
$$

Now, define the feasible assignment $\bar{A}$ as follows: for each $(i, j, k) \in \mathcal{B} \times \mathcal{G} \times \mathcal{S}$,

$$
\bar{A}_{i j k}= \begin{cases}0 & \text { if either }\{i, k\} \subset \mathcal{C} \text { or }\{i, k\} \subset \mathcal{C}^{c} \\ \widehat{A}_{i j k} & \text { otherwise, }\end{cases}
$$

where $\mathcal{C}^{c}$ is the complementary set of $\mathcal{C}$. Define a new market $\bar{M}=(\mathcal{B}, \mathcal{S}, \mathcal{G}, V, \bar{d}, R, \bar{Q})$, where the new vector of maximal demands $\bar{d}$ is defined by setting

$$
\bar{d}_{i}=d_{i}-\sum_{(j, k) \in \mathcal{G} \times \mathcal{S}} \bar{A}_{i j k}
$$

for all $i \in \mathcal{B}$, and the new matrix of capacities $\bar{Q}$ is defined by setting

$$
\bar{q}_{j k}=q_{j k}-\sum_{i \in \mathcal{B}} \bar{A}_{i j k}
$$

for all $(j, k) \in \mathcal{G} \times \mathcal{S}$. Note that if $i \in \mathcal{C}$ then, $\bar{d}_{i}=d_{i}-\sum_{(j, k) \in \mathcal{G} \times\left(\mathcal{S}^{\mathcal{C}}\right)^{c}} \widehat{A}_{i j k}$ and if $k \in \mathcal{C}$ then, $\bar{q}_{j k}=q_{j k}-\sum_{i \in\left(\mathcal{B}^{c}\right)^{c}} \widehat{A}_{i j k}$ for all $j \in \mathcal{G}$.

\footnotetext{
${ }^{11} \phi^{M}(C, \widehat{A}, p)$ is obtained from $\phi^{M}(C, \widehat{A}, \Gamma)$ by replacing $\Gamma_{i j k}$ by $p_{j}$ for all $(i, j, k) \in B \times G \times S$.
} 
By (9),

$$
\sum_{i \in \mathcal{B}^{\mathcal{C}}} u_{i}(p)+\sum_{k \in \mathcal{S}^{\mathcal{C}}} w_{k}(p)=\sum_{i \in \mathcal{B}^{\mathcal{C}}} d_{i} \cdot \gamma_{i}(p)+\sum_{(j, k) \in \mathcal{G} \times \mathcal{S}^{\mathcal{C}}} q_{j k} \cdot \pi_{j k}(p)
$$

and note that

$$
\begin{aligned}
\sum_{i \in \mathcal{B}^{\mathcal{C}}} d_{i} \cdot \gamma_{i}(p) & =\sum_{i \in \mathcal{B}^{\mathcal{C}}} \bar{d}_{i} \cdot \gamma_{i}(p)+\sum_{i \in \mathcal{B}^{\mathcal{C}}}\left(\sum_{(j, k) \in \mathcal{G} \times \mathcal{S}} \bar{A}_{i j k}\right) \cdot \gamma_{i}(p) \\
& =\sum_{i \in \mathcal{B}^{\mathcal{C}}} \bar{d}_{i} \cdot \gamma_{i}(p)+\sum_{i \in \mathcal{B}^{\mathcal{C}}}\left(\sum_{j \in \mathcal{G}} \sum_{k \in\left(\mathcal{S}^{\mathcal{C}}\right)^{c}} \widehat{A}_{i j k}\right) \cdot \gamma_{i}(p)
\end{aligned}
$$

and for each $j \in \mathcal{G}$,

$$
\begin{aligned}
\sum_{k \in \mathcal{S}^{\mathcal{C}}} q_{j k} \cdot \pi_{j k}(p) & =\sum_{k \in \mathcal{S}^{\mathcal{C}}} \bar{q}_{j k} \cdot \pi_{j k}(p)+\sum_{k \in \mathcal{S}^{\mathcal{C}}}\left(\sum_{i \in \mathcal{B}} \bar{A}_{i j k}\right) \cdot \pi_{j k}(p) \\
& =\sum_{k \in \mathcal{S}^{\mathcal{C}}} \bar{q}_{j k} \cdot \pi_{j k}(p)+\sum_{k \in \mathcal{S}^{\mathcal{C}}}\left(\sum_{i \in\left(\mathcal{B}^{\mathcal{C}}\right)^{c}} \widehat{A}_{i j k}\right) \cdot \pi_{j k}(p)
\end{aligned}
$$

Hence,

$$
\begin{aligned}
\sum_{i \in \mathcal{B}^{\mathcal{C}}} u_{i}(p)+\sum_{k \in \mathcal{S}^{\mathcal{C}}} w_{k}(p)=\sum_{i \in \mathcal{B}^{\mathcal{C}}} \bar{d}_{i} \cdot \gamma_{i}(p)+\sum_{i \in \mathcal{B}^{\mathcal{C}}}\left(\sum_{j \in \mathcal{G}} \sum_{k \in\left(\mathcal{S}^{\mathcal{C}}\right)^{c}} \widehat{A}_{i j k}\right) \cdot \gamma_{i}(p) \\
+\sum_{k \in \mathcal{S}^{\mathcal{C}}} \sum_{j \in \mathcal{G}} \bar{q}_{j k} \cdot \pi_{j k}(p)+\sum_{k \in \mathcal{S}^{\mathcal{C}}} \sum_{j \in \mathcal{G}}\left(\sum_{i \in\left(\mathcal{B}^{\mathcal{C}}\right)^{c}} \widehat{A}_{i j k}\right) \cdot \pi_{j k}(p) .
\end{aligned}
$$

By (7), for every $i \in \mathcal{B}$ and $j \in \mathcal{G}$,

$$
\gamma_{i}(p) \geq v_{i j}-p_{j}
$$

Moreover, by (8), for every $(j, k) \in \mathcal{G} \times \mathcal{S}$,

$$
\pi_{j k}(p) \geq p_{j}-r_{j k}
$$

By (A.3) and (A.4)

$$
\begin{aligned}
\sum_{(i, j, k) \in \mathcal{B}^{\mathcal{C}} \times \mathcal{G} \times \mathcal{S}^{\mathcal{C}}} \tau_{i j k} \cdot \widehat{A}_{i j k}+\sum_{(i, j, k) \in \mathcal{B}^{\mathcal{C}} \times \mathcal{G} \times\left(\mathcal{S}^{\mathcal{C}}\right)^{c}}\left(v_{i j}-p_{j}\right) \cdot \widehat{A}_{i j k}+ \\
\sum_{(i, j, k) \in\left(\mathcal{B}^{\mathcal{C}}\right)^{c} \times \mathcal{G} \times \mathcal{S}^{\mathcal{C}}}\left(p_{j}-r_{j k}\right) \cdot \widehat{A}_{i j k}>\sum_{i \in \mathcal{B}^{\mathcal{C}}} \bar{d}_{i} \cdot \gamma_{i}(p)+\sum_{i \in \mathcal{B}^{\mathcal{C}}}\left(\sum_{j \in \mathcal{G}} \sum_{k \in\left(\mathcal{S}^{\mathcal{C}}\right)^{c}} \widehat{A}_{i j k}\right) \cdot \gamma_{i}(p) \\
\quad+\sum_{k \in \mathcal{S}^{\mathcal{C}}} \sum_{j \in \mathcal{G}} \bar{q}_{j k} \cdot \pi_{j k}(p)+\sum_{k \in \mathcal{S}^{\mathcal{C}}} \sum_{j \in \mathcal{G}}\left(\sum_{i \in\left(\mathcal{B}^{\mathcal{C}}\right)^{c}} \widehat{A}_{i j k}\right) \cdot \pi_{j k}(p) .
\end{aligned}
$$


By (A.5),

$$
\sum_{i \in \mathcal{B}^{\mathcal{C}}}\left(\sum_{j \in \mathcal{G}} \sum_{k \in\left(\mathcal{S}^{\mathcal{C}}\right)^{c}} \widehat{A}_{i j k}\right) \cdot \gamma_{i}(p) \geq \sum_{(i, j, k) \in \mathcal{B}^{\mathcal{C}} \times \mathcal{G} \times\left(\mathcal{S}^{\mathcal{C}}\right)^{c}}\left(v_{i j}-p_{j}\right) \cdot \widehat{A}_{i j k} .
$$

Hence, by (A.7),

$$
\begin{aligned}
\sum_{(i, j, k) \in \mathcal{B}^{\mathcal{C}} \times \mathcal{G} \times \mathcal{S}^{\mathcal{C}}} \tau_{i j k} \cdot \widehat{A}_{i j k}+ & \sum_{(i, j, k) \in\left(\mathcal{B}^{\mathcal{C}}\right)^{c} \times \mathcal{G} \times \mathcal{S}^{\mathcal{C}}}\left(p_{j}-r_{j k}\right) \cdot \widehat{A}_{i j k}> \\
& \sum_{i \in \mathcal{B}^{\mathcal{C}}} \bar{d}_{i} \cdot \gamma_{i}(p)+\sum_{k \in \mathcal{S}^{\mathcal{C}}} \sum_{j \in \mathcal{G}} \bar{q}_{j k} \cdot \pi_{j k}(p) \\
& +\sum_{k \in \mathcal{S}^{\mathcal{C}}} \sum_{j \in \mathcal{G}}\left(\sum_{i \in\left(\mathcal{B}^{\mathcal{C}}\right)^{c}} \widehat{A}_{i j k}\right) \cdot \pi_{j k}(p) .
\end{aligned}
$$

By (A.6),

$$
\sum_{k \in \mathcal{S}^{\mathcal{C}}} \sum_{j \in \mathcal{G}}\left(\sum_{i \in\left(\mathcal{B}^{\mathcal{C}}\right)^{c}} \widehat{A}_{i j k}\right) \cdot \pi_{j k}(p) \geq \sum_{(i, j, k) \in\left(\mathcal{B}^{\mathcal{C}}\right)^{c} \times \mathcal{G} \times \mathcal{S}^{\mathcal{C}}}\left(p_{j}-r_{j k}\right) \cdot \widehat{A}_{i j k} .
$$

Hence, by (A.8),

$$
\sum_{(i, j, k) \in \mathcal{B}^{\mathcal{C}} \times \mathcal{G} \times \mathcal{S}^{\mathcal{C}}} \tau_{i j k} \cdot \widehat{A}_{i j k}>\sum_{i \in \mathcal{B}^{\mathcal{C}}} \bar{d}_{i} \cdot \gamma_{i}(p)+\sum_{k \in \mathcal{S}^{\mathcal{C}}} \sum_{j \in \mathcal{G}} \bar{q}_{j k} \cdot \pi_{j k}(p) .
$$

Observe that since $\widehat{A}$ is a feasible assignment,

$$
v^{\bar{M}}(\mathcal{C}) \geq \sum_{(i, j, k) \in \mathcal{B}^{\mathcal{C}} \times \mathcal{G} \times \mathcal{S}^{\mathcal{C}}} \tau_{i j k} \cdot \widehat{A}_{i j k}
$$

where $v^{\bar{M}}(\mathcal{C})=T^{\bar{M}}\left(A^{\mathcal{C}}\right)$ for any optimal assignment $A^{\mathcal{C}}$ of market $\bar{M}$. Since $(\gamma(p), \pi(p)) \in D$ then by (A.1), $\left(\gamma^{\mathcal{C}}(p), \pi^{\mathcal{C}}(p)\right) \in D^{\mathcal{C}}$ for market $\bar{M}$. Hence, by the Strong Duality Theorem,

$$
\sum_{i \in \mathcal{B}^{\mathcal{C}}} \bar{d}_{i} \cdot \gamma_{i}(p)+\sum_{k \in \mathcal{S}^{\mathcal{C}}} \sum_{j \in \mathcal{G}} \bar{q}_{j k} \cdot \pi_{j k}(p)=v^{\bar{M}}(\mathcal{C}),
$$

contradicting (A.9).

Lemmas 1 to 6 below will be used to prove Theorem 1 .

Lemma 1 Let $M=(\mathcal{B}, \mathcal{G}, \mathcal{S}, V, d, R, Q)$ be a market and let $\rho \geq 2$. Then

$$
\mathcal{C} o^{\rho M} \subset\left\{\left(u^{\rho}, w^{\rho}\right) \equiv(\underbrace{(u, w), \ldots,(u, w)}_{\rho \text {-times }}) \in\left(\mathbb{R}^{B_{1}} \times \mathbb{R}^{S_{1}}\right) \times \ldots \times\left(\mathbb{R}^{B_{\rho}} \times \mathbb{R}^{S_{\rho}}\right) \mid(u, w) \in \mathcal{C} o^{M}\right\} .
$$

Proof Let $\left(\left(\widehat{u}_{i_{1}}, \ldots, \widehat{u}_{i_{\rho}}\right)_{i \in \mathcal{B}},\left(\widehat{w}_{k_{1}}, \ldots, \widehat{w}_{k_{\rho}}\right)_{k \in \mathcal{S}}\right) \in \mathcal{C} o^{\rho M}$. For every $\alpha=1, \ldots, \rho$,

$$
\sum_{i_{\alpha} \in \mathcal{B}_{\alpha}} \widehat{u}_{i_{\alpha}}+\sum_{k_{\alpha} \in \mathcal{S}_{\alpha}} \widehat{w}_{k_{\alpha}} \geq v\left(\mathcal{B}_{\alpha} \cup \mathcal{S}_{\alpha}\right)
$$


must hold; otherwise, any coalition $\mathcal{C}=\mathcal{B}_{\alpha} \cup \mathcal{S}_{\alpha}$ would Core-block $(\widehat{u}, \widehat{w})$. Since for every $\alpha=1, \ldots, \rho, v\left(\mathcal{B}_{\alpha} \cup \mathcal{S}_{\alpha}\right)=v(\mathcal{B} \cup \mathcal{S})$,

$$
\sum_{i_{\alpha} \in \mathcal{B}_{\alpha}} \widehat{u}_{i_{\alpha}}+\sum_{k_{\alpha} \in \mathcal{S}_{\alpha}} \widehat{w}_{k_{\alpha}} \geq v(\mathcal{B} \cup \mathcal{S})
$$

must hold for every $\alpha=1, \ldots, \rho$. Let $A$ be an optimal assignment for market $M$. Define the assignment $A^{\rho}$ for market $\rho M$ as follows. For any $\left.\left(i_{\alpha}, j, k_{\beta}\right) \in\left(\mathcal{B}_{1} \cup \ldots \cup \mathcal{B}_{\rho}\right), \mathcal{G}, \mathcal{S}_{1} \cup \ldots \cup \mathcal{S}_{\rho}\right)$ set

$$
A_{i_{\alpha} j k_{\beta}}^{\rho}= \begin{cases}A_{i j k} & \text { if } \alpha=\beta \\ 0 & \text { otherwise. }\end{cases}
$$

Then, it is easy to show that $A^{\rho}$ is an optimal assignment for market $\rho M$ and $T^{\rho M}\left(A^{\rho}\right)=$ $\rho T^{M}(A)$. Since, by its definition, $v(\mathcal{B} \cup \mathcal{S})=T^{M}(A)$, (A.10) implies

$$
\sum_{\alpha=1}^{\rho}\left(\sum_{i_{\alpha} \in \mathcal{B}_{\alpha}} \widehat{u}_{i_{\alpha}}+\sum_{k_{\alpha} \in \mathcal{S}_{\alpha}} \widehat{w}_{k_{\alpha}}\right) \geq \rho v(\mathcal{B} \cup \mathcal{S})=\rho T^{M}(A)=T^{\rho M}\left(A^{\rho}\right) .
$$

Hence, since $A^{\rho}$ is an optimal assignment for market $\rho M$,

$$
\sum_{\alpha=1}^{\rho}\left(\sum_{i_{\alpha} \in \mathcal{B}_{\alpha}} \widehat{u}_{i_{\alpha}}+\sum_{k_{\alpha} \in \mathcal{S}_{\alpha}} \widehat{w}_{k_{\alpha}}\right)=T^{\rho M}\left(A^{\rho}\right) .
$$

Thus,

$$
\sum_{\alpha=1}^{\rho}\left(\sum_{i_{\alpha} \in \mathcal{B}_{\alpha}} \widehat{u}_{i_{\alpha}}+\sum_{k_{\alpha} \in \mathcal{S}_{\alpha}} \widehat{w}_{k_{\alpha}}\right)=\rho v(\mathcal{B} \cup \mathcal{S})
$$

By (A.10),

$$
\sum_{i_{\alpha} \in \mathcal{B}_{\alpha}} \widehat{u}_{i_{\alpha}}+\sum_{k_{\alpha} \in \mathcal{S}_{\alpha}} \widehat{w}_{k_{\alpha}}=v(\mathcal{B} \cup \mathcal{S})
$$

must hold for every $\alpha=1, \ldots, \rho$. Assume that there exists a buyer type $\hat{\imath} \in \mathcal{B}$ and two replicas $\alpha$ and $\alpha^{\prime}$ such that

$$
\widehat{u}_{\hat{\imath}_{\alpha}}>\widehat{u}_{\hat{\imath}_{\alpha^{\prime}}} .
$$

Then, the coalition $\mathcal{C}=\left[\left(\mathcal{B}_{\alpha} \cup \mathcal{S}_{\alpha}\right) \backslash\left\{\hat{\imath}_{\alpha}\right\}\right] \cup\left\{\hat{\imath}_{\alpha^{\prime}}\right\}$ Core-blocks $(\widehat{u}, \widehat{w})$ because

$$
v(\mathcal{C})=v(\mathcal{B} \cup \mathcal{S})>\sum_{i_{\alpha} \in \mathcal{B}_{\alpha} \backslash\left\{\hat{\imath}_{\alpha}\right\}} \widehat{u}_{i_{\alpha}}+\widehat{u}_{\hat{\nu}_{\alpha^{\prime}}}+\sum_{k_{\alpha} \in \mathcal{S}_{\alpha}} \widehat{w}_{k_{\alpha}} .
$$

Similarly for any seller type $\widehat{k} \in \mathcal{S}$. Thus, $\left(\left(\widehat{u}_{i_{1}}, \ldots, \widehat{u}_{i_{\rho}}\right)_{i \in \mathcal{B}},\left(\widehat{w}_{k_{1}}, \ldots, \widehat{w}_{k_{\rho}}\right)_{k \in \mathcal{S}}\right)=\left(u^{\rho}, w^{\rho}\right)$ for some payoff vector $(u, w) \in \mathbb{R}^{B} \times \mathbb{R}^{S}$ of market $M$. To obtain a contradiction, assume that 
$(u, w) \notin \mathcal{C} o^{M}$. Then, there exists a coalition $\mathcal{C}$ that Core-blocks $(u, w)$. But then, $\mathcal{C}$ also Core-blocks $(\widehat{u}, \widehat{w})$, a contradiction with $(\widehat{u}, \widehat{w}) \in \mathcal{C} O^{\rho M}$.

Lemma 2 Let $M=(\mathcal{B}, \mathcal{G}, \mathcal{S}, V, d, R, Q)$ be a market and let $\rho \geq 2$. Then

$\mathcal{S} \mathcal{W}^{\rho M} \subset\left\{\left(u^{\rho}, w^{\rho}\right) \equiv(\underbrace{(u, w), \ldots,(u, w)}_{\rho \text {-times }}) \in\left(\mathbb{R}^{B_{1}} \times \mathbb{R}^{S_{1}}\right) \times \ldots \times\left(\mathbb{R}^{B_{\rho}} \times \mathbb{R}^{S_{\rho}}\right) \mid(u, w) \in \mathcal{S} \mathcal{W}^{M}\right\}$.

Proof Let $(\widehat{u}, \widehat{w}) \equiv\left(\left(\widehat{u}_{i_{1}}, \ldots, \widehat{u}_{i_{\rho}}\right)_{i \in \mathcal{B}},\left(\widehat{w}_{k_{1}}, \ldots, \widehat{w}_{k_{\rho}}\right)_{k \in \mathcal{S}}\right) \in \mathcal{S} \mathcal{W}^{\rho M}$. First, observe that for all $\alpha=1, \ldots, \rho,\left(\left(\widehat{u}_{i_{\alpha}}\right)_{i \in \mathcal{B}},\left(\widehat{w}_{k_{\alpha}}\right)_{k \in \mathcal{S}}\right) \in \mathcal{S} \mathcal{W}^{M}$. By Remark $3,\left(\left(\widehat{u}_{i_{\alpha}}\right)_{i \in \mathcal{B}},\left(\widehat{w}_{k_{\alpha}}\right)_{k \in \mathcal{S}}\right) \in \mathcal{C} o^{M}$. By Lemma 1 , for all $\alpha, \alpha^{\prime}=1, \ldots, \rho, \widehat{u}_{i_{\alpha}}=\widehat{u}_{i_{\alpha^{\prime}}}$ for all $i \in \mathcal{B}$ and $\widehat{w}_{k_{\alpha}}=\widehat{w}_{k_{\alpha^{\prime}}}$ for all $k \in \mathcal{S}$.

Lemma 3 Let $M=(\mathcal{B}, \mathcal{G}, \mathcal{S}, V, d, R, Q)$ be a market and let $(u, w) \in \mathcal{S} \mathcal{W}$. Then, there exists a distribution matrix $\Gamma=\left(\Gamma_{i j k}\right)_{(i, j, k) \in \mathcal{B} \times \mathcal{G} \times \mathcal{S}}$ such that for any optimal assignment $A$, and for every $i \in \mathcal{B}$ and $k \in \mathcal{S}$,

$$
u_{i}=\sum_{(j, k) \in \mathcal{G} \times \mathcal{S}}\left(v_{i j}-\Gamma_{i j k}\right) \cdot A_{i j k}
$$

and

$$
w_{k}=\sum_{(i, j) \in \mathcal{B} \times \mathcal{G}}\left(\Gamma_{i j k}-r_{j k}\right) \cdot A_{i j k} .
$$

Proof Assume $(u, w) \in \mathcal{S} \mathcal{W}$. By the definition of set-wise stability, there exists a distri-

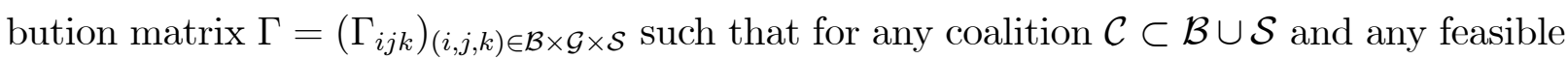
assignment $\widehat{A}$ that is $S W$-compatible with $\mathcal{C}$, we have that

$$
\sum_{i \in \mathcal{B}^{\mathcal{C}}} u_{i}+\sum_{k \in \mathcal{S}^{\mathcal{C}}} w_{i} \geq \phi^{M}(\mathcal{C}, \widehat{A}, \Gamma) .
$$

Let $A$ be any optimal assignment and consider either $\mathcal{C}=\{i\}$ or $\mathcal{C}=\{k\}$. Observe that $A$ itself is $S W$-compatible with $\mathcal{C}$. Then, by (A.11),

$$
u_{i} \geq \sum_{(j, k) \in \mathcal{G} \times \mathcal{S}}\left(v_{i j}-\Gamma_{i j k}\right) \cdot A_{i j k} \text { for every } i \in \mathcal{B}
$$

and

$$
w_{k} \geq \sum_{(i, j) \in \mathcal{B} \times \mathcal{G}}\left(\Gamma_{i j k}-r_{j k}\right) \cdot A_{i j k} \text { for every } k \in \mathcal{S}
$$

Since

$\sum_{i \in \mathcal{B}} \sum_{(j, k) \in \mathcal{G} \times \mathcal{S}}\left(v_{i j}-\Gamma_{i j k}\right) \cdot A_{i j k}+\sum_{k \in \mathcal{S}} \sum_{(i, j) \in \mathcal{B} \times \mathcal{G}}\left(\Gamma_{i j k}-r_{j k}\right) \cdot A_{i j k}=\sum_{(i, j, k) \in \mathcal{B} \times \mathcal{G} \times \mathcal{S}} \tau_{i j k} \cdot A_{i j k}=T^{M}(A)$ 
and

$$
T^{M}(A)=\sum_{i \in \mathcal{B}} u_{i}+\sum_{k \in \mathcal{S}} w_{k}
$$

the statement of Lemma 3 follows.

Lemma $4^{\prime}$ Let $M=(\mathcal{B}, \mathcal{G}, \mathcal{S}, V, d, R, Q)$ be a market and let $((u, w),(u, w)) \in \mathcal{S} \mathcal{W}^{2 M}$. Assume $A$ is an optimal assignment for market $M$ such that $A_{i j^{\prime} k} \neq 0$ for some $i \in \mathcal{B}$, $j^{\prime} \in \mathcal{G}$ and $k \in \mathcal{S}$. Let $\Gamma=\left(\Gamma_{i j k}\right)_{(i, j, k) \in\left(\mathcal{B}_{1} \cup \mathcal{B}_{2}\right) \times \mathcal{G} \times\left(\mathcal{S}_{1} \cup \mathcal{S}_{2}\right)}$ be any distribution matrix for market $2 M$ such that for any coalition $\mathcal{C} \subset \mathcal{B}_{1} \cup \mathcal{B}_{2} \cup \mathcal{S}_{1} \cup \mathcal{S}_{2}$ and any feasible assignment $\widehat{A}$ that is $S W$-compatible with $\mathcal{C}$ we have that

$$
\sum_{i \in \mathcal{C}} u_{i}+\sum_{k \in \mathcal{C}} w_{k} \geq \phi^{2 M}(\mathcal{C}, \widehat{A}, \Gamma)
$$

Then, $\Gamma_{i_{1} j^{\prime} k_{1}}=\Gamma_{i_{2} j^{\prime} k_{2}}$.

Proof Assume otherwise; for instance, $\Gamma_{i_{1} j^{\prime} k_{1}}>\Gamma_{i_{2} j^{\prime} k_{2}}$. If $\Gamma_{i_{1 j^{\prime} k_{1}}}<\Gamma_{i_{2} j^{\prime} k_{2}}$, then replace in the argument that follows the roles of $i_{1}$ and $k_{1}$ by $i_{2}$ and $k_{2}$, respectively. Consider the coalition $\mathcal{C}=\left\{i_{1}, k_{2}\right\}$. From the optimal assignment $A$ of market $M$ we define the assignment $\widehat{A}$ for market $2 M$ by decreasing in 1 unit the exchanges of good $j^{\prime}$ between $i_{1}$ and $k_{1}$ and between $i_{2}$ and $k_{2}$ and by simultaneously increasing in 1 unit of good $j^{\prime}$ the exchange between $i_{1}$ and $k_{2}$. Namely, for every $(i, j, k) \in \mathcal{B}_{1} \cup \mathcal{B}_{2} \times \mathcal{G} \times \mathcal{S}_{1} \cup \mathcal{S}_{2}$, define

$$
\widehat{A}_{i j k}= \begin{cases}A_{i j k}-1 & \text { if } i=i_{1}, j=j^{\prime} \text { and } k=k_{1} \\ A_{i j k}-1 & \text { if } i=i_{2}, j=j^{\prime} \text { and } k=k_{2} \\ A_{i j k}+1 & \text { if } i=i_{1}, j=j^{\prime} \text { and } k=k_{2} \\ A_{i j k} & \text { otherwise. }\end{cases}
$$

Observe that since by assumption $A_{i_{1} j^{\prime} k_{1}}=A_{i_{2} j^{\prime} k_{2}} \neq 0, \widehat{A}$ is a feasible assignment for market $2 M$. Moreover, $\widehat{A}$ is $S W$-compatible with $\mathcal{C}=\left\{i_{1}, k_{2}\right\}$. Define

$$
\begin{aligned}
\widehat{u}_{i_{1}}= & \sum_{(j, k) \in \mathcal{G} \times\left(\mathcal{S}_{1} \cup \mathcal{S}_{2}\right)}\left(v_{i_{1} j}-\Gamma_{i_{1} j k}\right) \cdot \widehat{A}_{i_{1} j k}=\sum_{(j, k) \in \mathcal{G} \times\left(\mathcal{S}_{1} \cup \mathcal{S}_{2}\right) \backslash\left\{k_{1}, k_{2}\right\}}\left(v_{i_{1} j}-\Gamma_{i_{1} j k}\right) \cdot A_{i_{1} j k} \\
& +\sum_{j \in \mathcal{G} \backslash\left\{j^{\prime}\right\}}\left(v_{i_{1} j}-\Gamma_{i_{1} j k_{1}}\right) \cdot A_{i_{1} j k_{1}}+\sum_{j \in \mathcal{G} \backslash\left\{j^{\prime}\right\}}\left(v_{i_{1} j}-\Gamma_{i_{1} j k_{2}}\right) \cdot A_{i_{1} j k_{2}} \\
& +\left(v_{i_{1} j^{\prime}}-\Gamma_{i_{1} j^{\prime} k_{1}}\right) \cdot\left(A_{i_{1} j^{\prime} k_{1}}-1\right)+\left(v_{i_{1} j^{\prime}}-\Gamma_{i_{1} j^{\prime} k_{2}}\right) \cdot\left(A_{i_{1} j^{\prime} k_{2}}+1\right)
\end{aligned}
$$


and

$$
\begin{aligned}
\widehat{w}_{k_{2}}=\sum_{(i, j) \in\left(\mathcal{B}_{1} \cup \mathcal{B}_{2}\right) \times \mathcal{G}}\left(\Gamma_{i j k_{2}}-r_{j k_{2}}\right) \cdot \widehat{A}_{i j k_{2}}=\sum_{(i, j) \in\left(\mathcal{B}_{1} \cup \mathcal{B}_{2}\right) \backslash\left\{i_{1}, i_{2}\right\} \times \mathcal{G}}\left(\Gamma_{i j k_{2}}-r_{j k_{2}}\right) \cdot A_{i j k_{2}} \\
\quad+\sum_{j \in \mathcal{G} \backslash\left\{j^{\prime}\right\}}\left(\Gamma_{i_{1} j k_{1}}-r_{j k_{2}}\right) \cdot A_{i_{1} j k_{2}}+\sum_{j \in \mathcal{G} \backslash\left\{j^{\prime}\right\}}\left(\Gamma_{i_{2} j k_{2}}-r_{j k_{2}}\right) \cdot A_{i_{2} j k_{2}} \\
\quad+\left(\Gamma_{i_{1} j^{\prime} k_{2}}-r_{j^{\prime} k_{2}}\right) \cdot\left(A_{i_{1} j^{\prime} k_{2}}+1\right)+\left(\Gamma_{i_{2} j^{\prime} k_{2}}-r_{j^{\prime} k_{2}}\right) \cdot\left(A_{i_{2} j^{\prime} k_{2}}-1\right) .
\end{aligned}
$$

On the other hand,

$$
\begin{aligned}
u_{i_{1}}= & \sum_{(j, k) \in \mathcal{G} \times\left(\mathcal{S}_{1} \cup \mathcal{S}_{2}\right)}\left(v_{i_{1} j}-\Gamma_{i_{1} j k}\right) \cdot A_{i_{1} j k}=\sum_{(j, k) \in \mathcal{G} \times\left(\mathcal{S}_{1} \cup \mathcal{S}_{2}\right) \backslash\left\{k_{1}, k_{2}\right\}}\left(v_{i_{1} j}-\Gamma_{i_{1} j k}\right) \cdot A_{i_{1} j k} \\
& +\sum_{j \in \mathcal{G} \backslash\left\{j^{\prime}\right\}}\left(v_{i_{1} j}-\Gamma_{i_{1} j k_{1}}\right) \cdot A_{i_{1} j k_{1}}+\sum_{j \in \mathcal{G} \backslash\left\{j^{\prime}\right\}}\left(v_{i_{1} j}-\Gamma_{i_{1} j k_{2}}\right) \cdot A_{i_{1} j k_{2}} \\
& +\left(v_{i_{1} j^{\prime}}-\Gamma_{i_{1} j^{\prime} k_{1}}\right) \cdot A_{i_{1} j^{\prime} k_{1}}+\left(v_{i_{1} j^{\prime}}-\Gamma_{i_{1} j^{\prime} k_{2}}\right) \cdot A_{i_{1} j^{\prime} k_{2}}
\end{aligned}
$$

and

$$
\begin{aligned}
w_{k_{2}}= & \sum_{(i, j) \in\left(\mathcal{B}_{1} \cup \mathcal{B}_{2}\right) \times \mathcal{G}}\left(\Gamma_{i j k_{2}}-r_{j k_{2}}\right) \cdot A_{i j k_{2}}=\sum_{(i, j) \in\left(\mathcal{B}_{1} \cup \mathcal{B}_{2}\right) \backslash\left\{i_{1}, i_{2}\right\} \times \mathcal{G}}\left(\Gamma_{i j k_{2}}-r_{j k_{2}}\right) \cdot A_{i j k_{2}} \\
& +\sum_{j \in \mathcal{G} \backslash\left\{j^{\prime}\right\}}\left(\Gamma_{i_{1} j k_{2}}-r_{j k_{2}}\right) \cdot A_{i_{1} j k_{2}}+\sum_{j \in \mathcal{G} \backslash\left\{j^{\prime}\right\}}\left(\Gamma_{i_{2} j k_{2}}-r_{j k_{2}}\right) \cdot A_{i_{2} j k_{2}} \\
& +\left(\Gamma_{i_{1} j^{\prime} k_{2}}-r_{j^{\prime} k_{2}}\right) \cdot A_{i_{1} j^{\prime} k_{2}}+\left(\Gamma_{i_{2} j^{\prime} k_{2}}-r_{j^{\prime} k_{2}}\right) \cdot A_{i_{2} j^{\prime} k_{2}} .
\end{aligned}
$$

By definition of $\widehat{A}$,

$$
\begin{aligned}
u_{i_{1}}+w_{k_{2}}-\left(\widehat{u}_{i_{1}}+\widehat{w}_{k_{2}}\right)=( & \left.v_{i_{1} j^{\prime}}-\Gamma_{i_{1} j^{\prime} k_{1}}\right) \cdot A_{i_{1} j^{\prime} k_{1}}+\left(v_{i_{1} j^{\prime}}-\Gamma_{i_{1} j^{\prime} k_{2}}\right) \cdot A_{i_{1} j^{\prime} k_{2}} \\
& +\left(\Gamma_{i_{1} j^{\prime} k_{2}}-r_{j^{\prime} k_{2}}\right) \cdot A_{i_{1} j^{\prime} k_{2}}+\left(\Gamma_{i_{2} j^{\prime} k_{2}}-r_{j^{\prime} k_{2}}\right) \cdot A_{i_{2} j^{\prime} k_{2}} \\
& -\left(v_{i_{1} j^{\prime}}-\Gamma_{i_{1} j^{\prime} k_{1}}\right) \cdot\left(A_{i_{1} j^{\prime} k_{1}}-1\right)-\left(v_{i_{1} j^{\prime}}-\Gamma_{i_{1} j^{\prime} k_{2}}\right) \cdot\left(A_{i_{1} j^{\prime} k_{2}}+1\right) \\
& -\left(\Gamma_{i_{1} j^{\prime} k_{2}}-r_{j^{\prime} k_{2}}\right) \cdot\left(A_{i_{1} j^{\prime} k_{2}}+1\right)-\left(\Gamma_{i_{2} j^{\prime} k_{2}}-r_{j^{\prime} k_{2}}\right) \cdot\left(A_{i_{2} j^{\prime} k_{2}}-1\right)= \\
& =\left(v_{i_{1} j^{\prime}}-\Gamma_{i_{1} j^{\prime} k_{1}}\right)-\left(v_{i_{1} j^{\prime}}-\Gamma_{i_{1} j^{\prime} k_{2}}\right) \\
& -\left(\Gamma_{i_{1} j^{\prime} k_{2}}-r_{j^{\prime} k_{2}}\right)+\left(\Gamma_{i_{2} j^{\prime} k_{2}}-r_{j^{\prime} k_{2}}\right) \\
& =-\Gamma_{i_{1} j^{\prime} k_{1}}+\Gamma_{i_{2} j^{\prime} k_{2}} .
\end{aligned}
$$

Since by assumption $\Gamma_{i_{1} j^{\prime} k_{1}}>\Gamma_{i_{2} j^{\prime} k_{2}}$, we have that $u_{i_{1}}+w_{k_{2}}<\widehat{u}_{i_{1}}+\widehat{w}_{k_{2}}$, a contradiction with (A.12).

Lemma 4 Let $M=(\mathcal{B}, \mathcal{G}, \mathcal{S}, V, d, R, Q)$ be a market and let $((u, w),(u, w)) \in \mathcal{S W}^{2 M}$. Assume $A$ is an optimal assignment for market $M$ such that $A_{i j^{\prime} k} \neq 0 \neq A_{i^{\prime} j^{\prime} k^{\prime}}$ for some $i, i^{\prime} \in \mathcal{B}, j^{\prime} \in \mathcal{G}$ and $k, k^{\prime} \in \mathcal{S}$, where $i$ and $i^{\prime}$, as well as $k$ and $k^{\prime}$, may be the same agent. Let $\Gamma=\left(\Gamma_{i j k}\right)_{(i, j, k) \in\left(\mathcal{B}_{1} \cup \mathcal{B}_{2}\right) \times \mathcal{G} \times\left(\mathcal{S}_{1} \cup \mathcal{S}_{2}\right)}$ be any distribution matrix for market $2 M$ such that for 
any coalition $\mathcal{C} \subset \mathcal{B}_{1} \cup \mathcal{B}_{2} \cup \mathcal{S}_{1} \cup \mathcal{S}_{2}$ and any feasible assignment $\widehat{A}$ that is $S W$-compatible with $\mathcal{C}$ we have that

$$
\sum_{i \in \mathcal{C}} u_{i}+\sum_{k \in \mathcal{C}} w_{k} \geq \phi^{2 M}(\mathcal{C}, \widehat{A}, \Gamma)
$$

Then, $\Gamma_{i j^{\prime} k}=\Gamma_{i^{\prime} j^{\prime} k^{\prime}}$.

Proof If $i=i^{\prime}$ and $k=k^{\prime}$ the result follows. Assume otherwise; for instance, $\Gamma_{i j^{\prime} k}>$ $\Gamma_{i^{\prime} j^{\prime} k^{\prime}}$. If $\Gamma_{i j^{\prime} k}<\Gamma_{i^{\prime} j^{\prime} k^{\prime}}$, then replace in the argument that follows the roles of $i$ and $k$ by $i^{\prime}$ and $k^{\prime}$, respectively. Consider the coalition $\mathcal{C}=\left\{i_{1}, k_{2}^{\prime}\right\}$. From the optimal assignment $A$ of market $M$ we define the assignment $\widehat{A}$ for market $2 M$ by decreasing in 1 unit the exchanges of good $j^{\prime}$ between $i_{1}$ and $k_{1}, i_{1}^{\prime}$ and $k_{1}^{\prime}$, and between $i_{2}^{\prime}$ and $k_{2}^{\prime}$ and by simultaneously increasing in 1 unit of good $j^{\prime}$ the exchange between $i_{1}$ and $k_{2}^{\prime}$. Namely, for every $(i, j, k) \in$ $\mathcal{B}_{1} \cup \mathcal{B}_{2} \times \mathcal{G} \times \mathcal{S}_{1} \cup \mathcal{S}_{2}$, define

$$
\widehat{A}_{i j k}=\left\{\begin{array}{cc}
A_{i j k}-1 & \text { if } i=i_{1}, j=j^{\prime} \text { and } k=k_{1} \\
A_{i j k}-1 & \text { if } i=i_{2}, j=j^{\prime} \text { and } k=k_{2} \\
A_{i j k}-1 & \text { if } i=i_{1}^{\prime}, j=j^{\prime} \text { and } k=k_{1}^{\prime} \\
A_{i j k}+1 & \text { if } i=i_{1}, j=j^{\prime} \text { and } k=k_{2}^{\prime} \\
A_{i j k} & \text { otherwise. }
\end{array}\right.
$$

Observe that since by assumption $A_{i j^{\prime} k} \neq 0 \neq A_{i^{\prime} j^{\prime} k^{\prime}}$, then $A_{i_{1} j^{\prime} k_{1}} \neq 0, A_{i_{2} j^{\prime} k_{2}} \neq 0, A_{i_{1}^{\prime} j^{\prime} k_{1}^{\prime}} \neq$ 0 and either $i \neq i^{\prime}$ or $k \neq k^{\prime}$ (or both) hold, $\widehat{A}$ is a feasible assignment for market $2 M$. Moreover, $\widehat{A}$ is $S W$-compatible with $\mathcal{C}=\left\{i_{1}, k_{2}^{\prime}\right\}$. Define

$$
\begin{aligned}
\widehat{u}_{i_{1}}= & \sum_{(j, k) \in \mathcal{G} \times\left(\mathcal{S}_{1} \cup \mathcal{S}_{2}\right)}\left(v_{i_{1} j}-\Gamma_{i_{1} j k}\right) \cdot \widehat{A}_{i_{1} j k}=\sum_{(j, k) \in \mathcal{G} \times\left(\mathcal{S}_{1} \cup \mathcal{S}_{2}\right) \backslash\left\{k_{1}, k_{2}^{\prime}\right\}}\left(v_{i_{1} j}-\Gamma_{i_{1} j k}\right) \cdot A_{i_{1} j k} \\
& +\sum_{j \in \mathcal{G} \backslash\left\{j^{\prime}\right\}}\left(v_{i_{1} j}-\Gamma_{i_{1} j k_{1}}\right) \cdot A_{i_{1} j k_{1}}+\sum_{j \in \mathcal{G} \backslash\left\{j^{\prime}\right\}}\left(v_{i_{1} j}-\Gamma_{i_{1} j k_{2}^{\prime}}\right) \cdot A_{i_{1} j k_{2}^{\prime}} \\
& +\left(v_{i_{1} j^{\prime}}-\Gamma_{i_{1} j^{\prime} k_{1}}\right) \cdot\left(A_{i_{1} j^{\prime} k_{1}}-1\right)+\left(v_{i_{1} j^{\prime}}-\Gamma_{i_{1} j^{\prime} k_{2}^{\prime}}\right) \cdot\left(A_{i_{1} j^{\prime} k_{2}^{\prime}}+1\right)
\end{aligned}
$$

and

$$
\begin{aligned}
\widehat{w}_{k_{2}^{\prime}}= & \sum_{(i, j) \in\left(\mathcal{B}_{1} \cup \mathcal{B}_{2}\right) \times \mathcal{G}}\left(\Gamma_{i j k_{2}^{\prime}}-r_{j k_{2}^{\prime}}\right) \cdot \widehat{A}_{i j k_{2}^{\prime}}=\sum_{(i, j) \in\left(\mathcal{B}_{1} \cup \mathcal{B}_{2}\right) \backslash\left\{i_{1}, i_{2}^{\prime}\right\} \times \mathcal{G}}\left(\Gamma_{i j k_{2}^{\prime}}-r_{j k_{2}^{\prime}}\right) \cdot A_{i j k_{2}^{\prime}} \\
& +\sum_{j \in \mathcal{G} \backslash\left\{j^{\prime}\right\}}\left(\Gamma_{i_{1} j k_{1}}-r_{j k_{2}^{\prime}}\right) \cdot A_{i_{1} j k_{2}^{\prime}}+\sum_{j \in \mathcal{G} \backslash\left\{j^{\prime}\right\}}\left(\Gamma_{i_{2}^{\prime} j k_{2}^{\prime}}-r_{j k_{2}^{\prime}}\right) \cdot A_{i_{2}^{\prime} j k_{2}^{\prime}} \\
& +\left(\Gamma_{i_{1} j^{\prime} k_{2}^{\prime}}-r_{j^{\prime} k_{2}^{\prime}}\right) \cdot\left(A_{i_{1} j^{\prime} k_{2}^{\prime}}+1\right)+\left(\Gamma_{i_{2}^{\prime} j^{\prime} k_{2}^{\prime}}-r_{j^{\prime} k_{2}^{\prime}}\right) \cdot\left(A_{i_{2}^{\prime} j^{\prime} k_{2}^{\prime}}-1\right) .
\end{aligned}
$$


On the other hand,

$$
\begin{aligned}
u_{i_{1}}= & \sum_{(j, k) \in \mathcal{G} \times\left(\mathcal{S}_{1} \cup \mathcal{S}_{2}\right)}\left(v_{i_{1} j}-\Gamma_{i_{1} j k}\right) \cdot A_{i_{1} j k}=\sum_{(j, k) \in \mathcal{G} \times\left(\mathcal{S}_{1} \cup \mathcal{S}_{2}\right) \backslash\left\{k_{1}, k_{2}^{\prime}\right\}}\left(v_{i_{1} j}-\Gamma_{i_{1} j k}\right) \cdot A_{i_{1} j k} \\
& +\sum_{j \in \mathcal{G} \backslash\left\{j^{\prime}\right\}}\left(v_{i_{1} j}-\Gamma_{i_{1} j k_{1}}\right) \cdot A_{i_{1} j k_{1}}+\sum_{j \in \mathcal{G} \backslash\left\{j^{\prime}\right\}}\left(v_{i_{1} j}-\Gamma_{i_{1} j k_{2}^{\prime}}\right) \cdot A_{i_{1} j k_{2}^{\prime}} \\
& +\left(v_{i_{1} j^{\prime}}-\Gamma_{i_{1} j^{\prime} k_{1}}\right) \cdot A_{i_{1} j^{\prime} k_{1}}+\left(v_{i_{1} j^{\prime}}-\Gamma_{i_{1} j^{\prime} k_{2}^{\prime}}\right) \cdot A_{i_{1} j^{\prime} k_{2}^{\prime}}
\end{aligned}
$$

and

$$
\begin{aligned}
w_{k_{2}^{\prime}}= & \sum_{(i, j) \in\left(\mathcal{B}_{1} \cup \mathcal{B}_{2}\right) \times \mathcal{G}}\left(\Gamma_{i j k_{2}^{\prime}}-r_{j k_{2}^{\prime}}\right) \cdot A_{i j k_{2}^{\prime}}=\sum_{(i, j) \in\left(\mathcal{B}_{1} \cup \mathcal{B}_{2}\right) \backslash\left\{i_{1}, i_{2}^{\prime}\right\} \times \mathcal{G}}\left(\Gamma_{i j k_{2}^{\prime}}-r_{j k_{2}^{\prime}}\right) \cdot A_{i j k_{2}^{\prime}} \\
& +\sum_{j \in \mathcal{G} \backslash\left\{j^{\prime}\right\}}\left(\Gamma_{i_{1} j k_{2}^{\prime}}-r_{j k_{2}^{\prime}}\right) \cdot A_{i_{1} j k_{2}^{\prime}}+\sum_{j \in \mathcal{G} \backslash\left\{j^{\prime}\right\}}\left(\Gamma_{i_{2}^{\prime} j k_{2}^{\prime}}-r_{j k_{2}^{\prime}}\right) \cdot A_{i_{2}^{\prime} j k_{2}^{\prime}} \\
& +\left(\Gamma_{i_{1} j^{\prime} k_{2}^{\prime}}-r_{j^{\prime} k_{2}^{\prime}}\right) \cdot A_{i_{1} j^{\prime} k_{2}^{\prime}}+\left(\Gamma_{i_{2}^{\prime} j^{\prime} k_{2}^{\prime}}-r_{j^{\prime} k_{2}^{\prime}}\right) \cdot A_{i_{2}^{\prime} j^{\prime} k_{2}^{\prime}} .
\end{aligned}
$$

By definition of $\widehat{A}$,

$$
\begin{aligned}
& u_{i_{1}}+w_{k_{2}^{\prime}}-\left(\widehat{u}_{i_{1}}+\widehat{w}_{k_{2}^{\prime}}\right)=\left(v_{i_{1} j^{\prime}}-\Gamma_{i_{1} j^{\prime} k_{1}}\right) \cdot A_{i_{1} j^{\prime} k_{1}}+\left(v_{i_{1} j^{\prime}}-\Gamma_{i_{1} j^{\prime} k_{2}^{\prime}}\right) \cdot A_{i_{1} j^{\prime} k_{2}^{\prime}} \\
& +\left(\Gamma_{i_{1} j^{\prime} k_{2}^{\prime}}-r_{j^{\prime} k_{2}^{\prime}}\right) \cdot A_{i_{1} j^{\prime} k_{2}^{\prime}}+\left(\Gamma_{i_{2}^{\prime} j^{\prime} k_{2}^{\prime}}-r_{j^{\prime} k_{2}^{\prime}}\right) \cdot A_{i_{2}^{\prime} j^{\prime} k_{2}^{\prime}} \\
& -\left(v_{i_{1} j^{\prime}}-\Gamma_{i_{1} j^{\prime} k_{1}}\right) \cdot\left(A_{i_{1} j^{\prime} k_{1}}-1\right)-\left(v_{i_{1} j^{\prime}}-\Gamma_{i_{1} j^{\prime} k_{2}^{\prime}}\right) \cdot\left(A_{i_{1} j^{\prime} k_{2}^{\prime}}+1\right) \\
& -\left(\Gamma_{i_{1} j^{\prime} k_{2}^{\prime}}-r_{j^{\prime} k_{2}^{\prime}}\right) \cdot\left(A_{i_{1} j^{\prime} k_{2}^{\prime}}+1\right)-\left(\Gamma_{i_{2}^{\prime} j^{\prime} k_{2}^{\prime}}-r_{j^{\prime} k_{2}^{\prime}}\right) \cdot\left(A_{i_{2}^{\prime} j^{\prime} k_{2}^{\prime}}-1\right) \text {. } \\
& =\left(v_{i_{1} j^{\prime}}-\Gamma_{i_{1} j^{\prime} k_{1}}\right)-\left(v_{i_{1} j^{\prime}}-\Gamma_{i_{1} j^{\prime} k_{2}^{\prime}}\right) \\
& -\left(\Gamma_{i_{1} j^{\prime} k_{2}^{\prime}}-r_{j^{\prime} k_{2}^{\prime}}\right)+\left(\Gamma_{i_{2}^{\prime} j^{\prime} k_{2}^{\prime}}-r_{j^{\prime} k_{2}^{\prime}}\right) \\
& =-\Gamma_{i_{1} j^{\prime} k_{1}}+\Gamma_{i_{2}^{\prime} j^{\prime} k_{2}^{\prime}} \text {. }
\end{aligned}
$$

Since by assumption $\Gamma_{i j^{\prime} k}>\Gamma_{i^{\prime} j^{\prime} k^{\prime}}$, by lemma $4^{\circ}$, we have that $\Gamma_{i_{1} j^{\prime} k_{1}}>\Gamma_{i_{2}^{\prime} j^{\prime} k_{2}^{\prime}}$, we have that $u_{i_{1}}+w_{k_{2}^{\prime}}<\widehat{u}_{i_{1}}+\widehat{w}_{k_{2}^{\prime}}$, a contradiction with (A.12).

Lemma 5 Let $M=(\mathcal{B}, \mathcal{G}, \mathcal{S}, V, d, R, Q)$ be a market and let $((u, w),(u, w)) \in \mathcal{S} \mathcal{W}^{2 M}$. Then, there exists a competitive equilibrium price $p=\left(p_{1}, \ldots, p_{G}\right)$, with $p_{j} \geq \min \left\{r_{j k} \mid k \in \mathcal{S}\right.$ such that $\left.q_{j k}>0\right\}$ for all $j \in \mathcal{G}$, such that for any coalition $\mathcal{C} \subset \mathcal{B} \cup \mathcal{S}$ and any feasible assignment $\widehat{A}$ that is $S W$-compatible with $\mathcal{C}$, we have that:

$$
\sum_{i \in \mathcal{B}^{\mathcal{C}}} u_{i}+\sum_{k \in \mathcal{S}^{\mathcal{C}}} w_{k} \geq \phi^{M}(\mathcal{C}, \widehat{A}, p) .
$$

Proof Assume that $((u, w),(u, w)) \in \mathcal{S} \mathcal{W}^{2 M}$. Then, there exists a distribution matrix $\Gamma=\left(\Gamma_{i j k}\right)_{(i, j, k) \in \mathcal{B}_{1} \cup \mathcal{B}_{2} \times \mathcal{G} \times \mathcal{S}_{1} \cup \mathcal{S}_{2}}$ such that for any a coalition $\mathcal{C} \subset \mathcal{B}_{1} \cup \mathcal{B}_{2} \cup \mathcal{S}_{1} \cup \mathcal{S}_{2}$, and any feasible assignment $\widehat{A}$ that is $S W$-compatible with $\mathcal{C}$, we have that

$$
\sum_{i_{\alpha} \in \mathcal{C}} u_{i_{\alpha}}+\sum_{k_{\beta} \in \mathcal{C}} w_{k_{\beta}} \geq \phi^{2 M}(\mathcal{C}, \widehat{A}, \Gamma) .
$$


To proceed with the proof we define a price vector $p=\left(p_{j}\right)_{j \in \mathcal{G}} \in \mathbb{R}_{+}^{G}$ as follows. Consider first any $j \in \mathcal{G}$ for which there exist $i \in \mathcal{B}$ and $k \in \mathcal{S}$ such that $A_{i j k} \neq 0$ for some optimal assignment $A$ for market $M$. Then, define $p_{j}=\Gamma_{i j k}$. Second, for any $j \in \mathcal{G}$ such that for all optimal assignments $A$ for market $M$ and all $i \in \mathcal{B}$ and $k \in \mathcal{S}, A_{i j k}=0$, define $p_{j}=\min \left\{r_{j k} \mid k \in \mathcal{S}\right.$ is such that $\left.q_{j k}>0\right\}$. Then, by Lemma $4, p$ is well-defined. Observe that for any optimal assignment $A$ of market $M$, the price vector $p=\left(p_{1}, \ldots, p_{G}\right)$ satisfies that for every coalition $\mathcal{C} \subset \mathcal{B} \cup \mathcal{S}$ and every feasible assignment $\widehat{A}$ that is $S W$-compatible with $\mathcal{C}$ we have that:

$$
\sum_{i \in \mathcal{B}^{\mathcal{C}}} u_{i}+\sum_{k \in \mathcal{S}^{\mathcal{C}}} w_{k} \geq \phi^{M}(\mathcal{C}, \widehat{A}, p) .
$$

We shall show that $(p, A)$ is a competitive equilibrium of $M$ by showing that the equilibrium conditions (E.D) and (E.S) are satisfied.

(E.D) For each buyer $i \in \mathcal{B}, A(i) \in D_{i}(p)$.

Since $A \in \mathcal{F}$, (D.a) and (D.b) hold.

(D.c): $\nabla_{i}^{>}(p) \neq \emptyset \Longrightarrow \sum_{j k} A(i)_{j k}=d_{i}$.

Assume $\nabla_{i^{\prime}}^{>}(p) \neq \emptyset$; i.e., there exists $j^{\prime} \in \mathcal{G}$ such that $v_{i^{\prime} j^{\prime}}-p_{j^{\prime}}=\max _{j \in \mathcal{G}}\left\{v_{i^{\prime} j}-p_{j}\right\}>0$. Assume that

$$
\sum_{j k} A_{i^{\prime} j k}<d_{i^{\prime}}
$$

Without loss of generality suppose that $i^{\prime}$ belongs to the first replica; i.e., $i^{\prime}=i_{1}$. Consider first the case where there are $i_{2} \in \mathcal{B}_{2}$ and $k_{2} \in \mathcal{S}_{2}$ with the property that $A_{i_{2} j^{\prime} k_{2}} \neq 0$. Consider the coalition $\mathcal{C}=\left\{i_{1}, k_{2}\right\}$ and its $S W$-compatible assignment $\widehat{A}$ where, for all $(i, j, k) \in \mathcal{B} \times \mathcal{G} \times \mathcal{S}$,

$$
\widehat{A}_{i j k}= \begin{cases}A_{i j k}+1 & \text { if } i=i_{1}, j=j^{\prime} \text { and } k=k_{2} \\ A_{i j k}-1 & \text { if } i=i_{2}, j=j^{\prime} \text { and } k=k_{2} \\ A_{i j k} & \text { otherwise. }\end{cases}
$$

By (A.15) and $A_{i_{2} j^{\prime} k_{2}} \neq 0, \widehat{A}$ is a feasible assignment and $S W$-compatible with coalition $\left\{i_{1}, k_{2}\right\}$. Then, define $\widehat{u}_{i_{1}}$ and $\widehat{w}_{k_{2}}$ as the payoffs of buyer $i_{1}$ and seller $k_{2}$ at assignment $\widehat{A}$, 
respectively. Then, by definition of $\widehat{A}$,

$$
\begin{aligned}
u_{i_{1}}+w_{k_{2}}-\left(\widehat{u}_{i_{1}}+\widehat{w}_{k_{2}}\right)=( & \left.v_{i_{1} j^{\prime}}-\Gamma_{i_{1} j^{\prime} k_{2}}\right) \cdot A_{i_{1} j^{\prime} k_{2}} \\
& +\left(\Gamma_{i_{1} j^{\prime} k_{2}}-r_{j^{\prime} k_{2}}\right) \cdot A_{i_{1} j^{\prime} k_{2}}+\left(\Gamma_{i_{2} j^{\prime} k_{2}}-r_{j^{\prime} k_{2}}\right) \cdot A_{i_{2} j^{\prime} k_{2}} \\
& -\left(v_{i_{1} j^{\prime}}-\Gamma_{i_{1} j^{\prime} k_{2}}\right) \cdot \widehat{A}_{i_{1} j^{\prime} k_{2}} \\
& -\left(\Gamma_{i_{1} j^{\prime} k_{2}}-r_{j^{\prime} k_{2}}\right) \cdot \widehat{A}_{i_{1} j^{\prime} k_{2}}-\left(\Gamma_{i_{2} j^{\prime} k_{2}}-r_{j^{\prime} k_{2}}\right) \cdot \widehat{A}_{i_{2} j^{\prime} k_{2}} \\
& =-\left(v_{i_{1} j^{\prime}}-\Gamma_{i_{1} j^{\prime} k_{2}}\right)-\left(\Gamma_{i_{1} j^{\prime} k_{2}}-r_{j^{\prime} k_{2}}\right)+\left(\Gamma_{i_{2} j^{\prime} k_{2}}-r_{j^{\prime} k_{2}}\right) \\
& =-v_{i_{1} j^{\prime}}+\Gamma_{i_{2} j^{\prime} k_{2}} .
\end{aligned}
$$

By Lemma $4, \Gamma_{i_{2} j^{\prime} k_{2}}=p_{j^{\prime}}$ and $A_{i_{2} j^{\prime} k_{2}} \neq 0$, by (A.14), $0 \geq v_{i_{1} j^{\prime}}-p_{j^{\prime}}$, which is a contradiction with $j^{\prime} \in \nabla_{i_{1}}^{>}(p)$.

Assume now that for all $i^{\prime \prime} \in \mathcal{B}_{1} \cup \mathcal{B}_{2}$ and all $k^{\prime \prime} \in \mathcal{S}_{1} \cup \mathcal{S}_{2}, A_{i^{\prime \prime} j^{\prime} k^{\prime \prime}}=0$. By definition, $p_{j^{\prime}}=\min \left\{r_{j^{\prime} k} \mid k\right.$ is such that $\left.q_{j^{\prime} k}>0\right\}$. Let $k^{*} \in \mathcal{S}_{1} \cup \mathcal{S}_{2}$ be such that $q_{j^{\prime} k^{*}}>0$. Consider the coalition $\mathcal{C}=\left\{i_{1}, k^{*}\right\}$ and its $S W$-compatible assignment $\widehat{A}$ where, for all $(i, j, k) \in\left(\mathcal{B}_{1} \cup \mathcal{B}_{2}\right) \times \mathcal{G} \times\left(\mathcal{S}_{1} \cup \mathcal{S}_{2}\right)$

$$
\widehat{A}_{i j k}= \begin{cases}A_{i j k}+1 & \text { if } i=i_{1}, j=j^{\prime} \text { and } k=k^{*} \\ A_{i j k} & \text { otherwise. }\end{cases}
$$

By (A.15) and $q_{j^{\prime} k^{*}}>0, \widehat{A}$ is a feasible assignment. Then, as before,

$$
\begin{aligned}
u_{i_{1}}+w_{k^{*}}-\left(\widehat{u}_{i_{1}}+\widehat{w}_{k^{*}}\right)=( & \left.v_{i_{1} j^{\prime}}-\Gamma_{i_{1} j^{\prime} k^{*}}\right) \cdot A_{i_{1} j^{\prime} k^{*}}+\left(\Gamma_{i_{1} j^{\prime} k^{*}}-r_{j^{\prime} k^{*}}\right) \cdot A_{i_{1} j^{\prime} k^{*}} \\
& -\left(v_{i_{1} j^{\prime}}-\Gamma_{i_{1} j^{\prime} k^{*}}\right) \cdot \widehat{A}_{i_{1} j^{\prime} k^{*}}-\left(\Gamma_{i_{1} j^{\prime} k^{*}}-r_{j^{\prime} k^{*}}\right) \cdot \widehat{A}_{i_{1} j^{\prime} k^{*}} \\
& =-\left(v_{i_{1} j^{\prime}}-\Gamma_{i_{1} j^{\prime} k^{*}}\right)-\left(\Gamma_{i_{1} j^{\prime} k^{*}}-r_{j^{\prime} k^{*}}\right) \\
& =-v_{i_{1} j^{\prime}}+r_{j^{\prime} k^{*}} .
\end{aligned}
$$

By (A.14), $v_{i_{1} j^{\prime}} \leq r_{j^{\prime} k^{*}}$ for every $i_{1}$, which implies that $v_{i_{1} j^{\prime}} \leq p_{j^{\prime}}$ contradicting that $j^{\prime} \in \nabla_{i_{1}}^{>}(p)$.

(D.d): $\sum_{k} A(i)_{j k}>0 \Longrightarrow j \in \nabla_{i}^{\geq}(p)$.

Assume otherwise; i.e., there exist $i^{\prime}, j^{\prime}, k^{\prime}$ such that $A_{i^{\prime} j^{\prime} k^{\prime}} \neq 0$ and $j^{\prime} \notin \nabla_{i^{\prime}}^{>}(p)$. We distinguish between the following two cases.

Case 1: $v_{i^{\prime} j^{\prime}}-p_{j^{\prime}}<0$. Consider the coalition $\mathcal{C}=\left\{i^{\prime}\right\}$ and its compatible assignment $\widehat{A}$ where

$$
\widehat{A}_{i j k}= \begin{cases}0 & \text { if } i=i^{\prime}, j=j^{\prime} \text { and } k=k^{\prime} \\ A_{i j k} & \text { otherwise. }\end{cases}
$$

Define $\widehat{u}_{i^{\prime}}$ as the utility of buyer $i^{\prime}$ at assignment $\widehat{A}$. Then, it is immediate to see that $u_{i^{\prime}}<\widehat{u}_{i^{\prime}}$, contradicting (A.14). 
Case 2: There exists $j^{\prime \prime} \in \nabla_{i^{\prime}}^{\geq}(p)$ such that

$$
\left(v_{i^{\prime} j^{\prime \prime}}-p_{j^{\prime \prime}}\right)>\left(v_{i^{\prime} j^{\prime}}-p_{j^{\prime}}\right) \geq 0
$$

Note that $A_{i^{\prime} j^{\prime} k^{\prime}} \neq 0$. Assume first that there exist $i^{\prime \prime} \in \mathcal{B}_{1} \cup \mathcal{B}_{2}$ and $k^{\prime \prime} \in \mathcal{S}_{1} \cup \mathcal{S}_{2}$ such that $A_{i^{\prime \prime} j^{\prime \prime} k^{\prime \prime}} \neq 0$. This imply that $\left(i^{\prime}, k^{\prime}\right) \in \mathcal{B}_{\alpha} \times \mathcal{S}_{\alpha}$ with $\alpha \in\{1,2\}$ and $\left(i^{\prime \prime}, k^{\prime \prime}\right) \in \mathcal{B}_{\beta} \times \mathcal{S}_{\beta}$ with $\beta \in\{1,2\}$. Consider the coalition $\mathcal{C}=\left\{i^{\prime}, k^{\prime \prime}\right\}$ and its $S W$-compatible assignment $\widehat{A}$ where, for all $(i, j, k) \in \mathcal{B} \times \mathcal{G} \times \mathcal{S}$,

$$
\widehat{A}_{i j k}= \begin{cases}A_{i j k}-1 & \text { if } i=i^{\prime}, j=j^{\prime} \text { and } k=k^{\prime} \\ A_{i j k}-1 & \text { if } i=i^{\prime \prime}, j=j^{\prime \prime} \text { and } k=k^{\prime \prime} \\ A_{i j k}+1 & \text { if } i=i^{\prime}, j=j^{\prime \prime} \text { and } k=k^{\prime \prime} \\ A_{i j k} & \text { otherwise. }\end{cases}
$$

Then, define $\widehat{u}_{i^{\prime}}$ and $\widehat{w}_{k^{\prime \prime}}$ as the payoffs of buyer $i^{\prime}$ and seller $k^{\prime \prime}$ at assignment $\widehat{A}$, respectively. Then, by definition of $\widehat{A}$,

$$
\begin{aligned}
& u_{i^{\prime}}+w_{k^{\prime \prime}}-\left(\widehat{u}_{i^{\prime}}+\widehat{w}_{k^{\prime \prime}}\right)=\left(v_{i^{\prime} j^{\prime}}-\Gamma_{i^{\prime} j^{\prime} k^{\prime}}\right) \cdot A_{i^{\prime} j^{\prime} k^{\prime}}+\left(v_{i^{\prime} j^{\prime \prime}}-\Gamma_{i^{\prime} j^{\prime \prime} k^{\prime \prime}}\right) \cdot A_{i^{\prime} j^{\prime \prime} k^{\prime \prime}} \\
& +\left(\Gamma_{i^{\prime} j^{\prime \prime} k^{\prime \prime}}-r_{j^{\prime \prime} k^{\prime \prime}}\right) \cdot A_{i^{\prime} j^{\prime \prime} k^{\prime \prime}}+\left(\Gamma_{i^{\prime \prime} j^{\prime \prime} k^{\prime \prime}}-r_{j^{\prime \prime} k^{\prime \prime}}\right) \cdot A_{i^{\prime \prime} j^{\prime \prime} k^{\prime \prime}} \\
& -\left(v_{i^{\prime} j^{\prime}}-\Gamma_{i^{\prime} j^{\prime} k^{\prime}}\right) \cdot \widehat{A}_{i^{\prime} j^{\prime} k^{\prime}}-\left(v_{i^{\prime} j^{\prime \prime}}-\Gamma_{i^{\prime} j^{\prime \prime} k^{\prime \prime}}\right) \cdot \widehat{A}_{i^{\prime} j^{\prime \prime} k^{\prime \prime}} \\
& -\left(\Gamma_{i^{\prime} j^{\prime \prime} k^{\prime \prime}}-r_{j^{\prime \prime} k^{\prime \prime}}\right) \cdot \widehat{A}_{i^{\prime} j^{\prime \prime} k^{\prime \prime}}-\left(\Gamma_{i^{\prime \prime} j^{\prime \prime} k^{\prime \prime}}-r_{j^{\prime \prime} k^{\prime \prime}}\right) \cdot \widehat{A}_{i^{\prime \prime} j^{\prime \prime} k^{\prime \prime}} \\
& =\left(v_{i^{\prime} j^{\prime}}-\Gamma_{i^{\prime} j^{\prime} k^{\prime}}\right)-\left(v_{i^{\prime} j^{\prime \prime}}-\Gamma_{i^{\prime} j^{\prime \prime} k^{\prime \prime}}\right)-\left(\Gamma_{i^{\prime} j^{\prime \prime} k^{\prime \prime}}-r_{j^{\prime \prime} k^{\prime \prime}}\right)+\left(\Gamma_{i^{\prime \prime} j^{\prime \prime} k^{\prime \prime}}-r_{j^{\prime \prime} k^{\prime \prime}}\right) \\
& =v_{i^{\prime} j^{\prime}}-\Gamma_{i^{\prime} j^{\prime} k^{\prime}}-v_{i^{\prime} j^{\prime \prime}}+\Gamma_{i^{\prime} j^{\prime \prime} k^{\prime \prime}}-\Gamma_{i^{\prime} j^{\prime \prime} k^{\prime \prime}}+r_{j^{\prime \prime} k^{\prime \prime}}+\Gamma_{i^{\prime \prime} j^{\prime \prime} k^{\prime \prime}}-r_{j^{\prime \prime} k^{\prime \prime}} \\
& =v_{i^{\prime} j^{\prime}}-\Gamma_{i^{\prime} j^{\prime} k^{\prime}}-\left(v_{i^{\prime} j^{\prime \prime}}-\Gamma_{i^{\prime \prime} j^{\prime \prime} k^{\prime \prime}}\right) \text {. }
\end{aligned}
$$

By Lemma $4 \Gamma_{i^{\prime} j^{\prime} k^{\prime}}=p_{j^{\prime}}$ and $\Gamma_{i^{\prime \prime} j^{\prime \prime} k^{\prime \prime}}=p_{j^{\prime \prime}}$, and by (A.16), $u_{i^{\prime}}+w_{k^{\prime \prime}}-\left(\widehat{u}_{i^{\prime}}+\widehat{w}_{k^{\prime \prime}}\right)=$ $\left.v_{i^{\prime} j^{\prime}}-p_{j^{\prime}}-v_{i^{\prime} j^{\prime \prime}}+p_{j^{\prime \prime}}\right)<0$, a contradiction with (A.14). Assume now that for all $i^{\prime \prime} \in \mathcal{B}_{1} \cup \mathcal{B}_{2}$ and all $k^{\prime \prime} \in \mathcal{S}_{1} \cup \mathcal{S}_{2}, A_{i^{\prime \prime} j^{\prime \prime} k^{\prime \prime}}=0$. Let $k^{*} \in \mathcal{S}_{1} \cup \mathcal{S}_{2}$ be such that $q_{j^{\prime \prime} k^{*}}>0$. By (1), such $k^{*}$ does exist. Consider the coalition $\mathcal{C}=\left\{i^{\prime}, k^{*}\right\}$ and its $S W$-compatible assignment $\widehat{A}$ where, for all $(i, j, k) \in \mathcal{B} \times \mathcal{G} \times \mathcal{S}$,

$$
\widehat{A}_{i j k}= \begin{cases}A_{i j k}-1 & \text { if } i=i^{\prime}, j=j^{\prime} \text { and } k=k^{\prime} \\ 1 & \text { if } i=i^{\prime}, j=j^{\prime \prime} \text { and } k=k^{*} \\ A_{i j k} & \text { otherwise. }\end{cases}
$$


Then, proceeding as before, define $\widehat{u}_{i^{\prime}}$ and $\widehat{w}_{k^{*}}$ as the payoffs of buyer $i^{\prime}$ and seller $k^{*}$ at assignment $\widehat{A}$, respectively. Then, by definition of $\widehat{A}$,

$$
\begin{aligned}
u_{i^{\prime}}+w_{k^{*}}-\left(\widehat{u}_{i^{\prime}}+\widehat{w}_{k^{*}}\right)= & \left(v_{i^{\prime} j^{\prime}}-\Gamma_{i^{\prime} j^{\prime} k^{\prime}}\right) \cdot A_{i^{\prime} j^{\prime} k^{\prime}}-\left(v_{i^{\prime} j^{\prime}}-\Gamma_{i^{\prime} j^{\prime} k^{\prime}}\right) \cdot \widehat{A}_{i^{\prime} j^{\prime} k^{\prime}} \\
& -\left(v_{i^{\prime} j^{\prime \prime}}-\Gamma_{i^{\prime} j^{\prime \prime} k^{*}}\right) \cdot \widehat{A}_{i^{\prime} j^{\prime \prime} k^{*}}-\left(\Gamma_{i^{\prime} j^{\prime \prime} k^{*}}-r_{j^{\prime \prime} k^{*}}\right) \cdot \widehat{A}_{i^{\prime} j^{\prime \prime} k^{*}} \\
= & \left(v_{i^{\prime} j^{\prime}}-\Gamma_{i^{\prime} j^{\prime} k^{\prime}}\right)-\left(v_{i^{\prime} j^{\prime \prime}}-\Gamma_{i^{\prime} j^{\prime \prime} k^{*}}\right)-\left(\Gamma_{i^{\prime} j^{\prime \prime} k^{*}}-r_{j^{\prime \prime} k^{*}}\right) \\
= & v_{i^{\prime} j^{\prime}}-\Gamma_{i^{\prime} j^{\prime} k^{\prime}}-\left(v_{i^{\prime} j^{\prime \prime}}-r_{j^{\prime \prime} k^{*}}\right) .
\end{aligned}
$$

By Lemma 4, $\Gamma_{i^{\prime} j^{\prime} k^{\prime}}=p_{j^{\prime}}$, by (A.14), $v_{i^{\prime} j^{\prime}}-p_{j^{\prime}} \geq v_{i^{\prime} j^{\prime \prime}}-r_{j^{\prime \prime} k^{*}}$ for every $k^{*}$. Because $p_{j} \geq \min _{k}\left(r_{j k}\right)$, we have that $v_{i^{\prime} j^{\prime}}-p_{j^{\prime}} \geq v_{i^{\prime} j^{\prime \prime}}-p_{j^{\prime \prime}}$, a contradiction with (A.16).

(E.S) For each good $j \in \mathcal{G}$ and each seller $k \in \mathcal{S}, \sum_{i} A_{i j k} \in \mathcal{S}_{j k}\left(p_{j}\right)$.

Fix $j^{\prime} \in \mathcal{G}$ and $k^{\prime} \in \mathcal{S}$. Assume first that $p_{j^{\prime}}<r_{j^{\prime} k^{\prime}}$. We want to show that $\sum_{i} A_{i j^{\prime} k^{\prime}}=$ 0. Suppose that $A_{i^{\prime} j^{\prime} k^{\prime}} \neq 0$. Consider the coalition $\mathcal{C}=\left\{k^{\prime}\right\}$ and its $S W$-compatible assignment $\widehat{A}$ where, for every $(i, j, k) \in \mathcal{B} \times \mathcal{G} \times \mathcal{S}$,

$$
\widehat{A}_{i j k}= \begin{cases}0 & \text { if } i=i^{\prime}, j=j^{\prime} \text { and } k=k^{\prime} \\ A_{i j k} & \text { otherwise. }\end{cases}
$$

Define $\widehat{w}_{k^{\prime}}$ as the utility of seller $k^{\prime}$ at assignment $\widehat{A}$. Then, it is immediate to see that $w_{k^{\prime}}<\widehat{w}_{k^{\prime}}$, contradicting (A.14).

Assume now that $p_{j^{\prime}}=r_{j^{\prime} k^{\prime}}$. We want to show that $0 \leq \sum_{i} A_{i j^{\prime} k^{\prime}} \leq q_{j^{\prime} k^{\prime}}$. But this holds because $A$ is a feasible assignment.

Finally, assume that $p_{j^{\prime}}>r_{j^{\prime} k^{\prime}}$. We want to show that $\sum_{i} A_{i j^{\prime} k^{\prime}}=q_{j^{\prime} k^{\prime}}$. Assume $\sum_{i} A_{i j^{\prime} k^{\prime}}<q_{j^{\prime} k^{\prime}}$. Hence,

$$
q_{j^{\prime} k^{\prime}}>0 .
$$

Consider first the case where there exist $i^{\prime} \in \mathcal{B}_{1} \cup \mathcal{B}_{2}$ and $k^{\prime \prime} \in \mathcal{S}_{1} \cup \mathcal{S}_{2}$ such that $A_{i^{\prime} j^{\prime} k^{\prime \prime}} \neq 0$. This imply that $\left(i^{\prime}, k^{\prime \prime}\right) \in \mathcal{B}_{\alpha} \times \mathcal{S}_{\alpha}$ with $\alpha \in\{1,2\}$. Consider the coalition $\mathcal{C}=\left\{i^{\prime}, k^{\prime}\right\}$ and its SW-compatible assignment $\widehat{A}$ where, for all $(i, j, k) \in \mathcal{B} \times \mathcal{G} \times \mathcal{S}$,

$$
\widehat{A}_{i j k}= \begin{cases}A_{i j k}-1 & \text { if } i=i^{\prime}, j=j^{\prime} \text { and } k=k^{\prime \prime} \\ A_{i j k}+1 & \text { if } i=i^{\prime}, j=j^{\prime} \text { and } k=k^{\prime} \\ A_{i j k} & \text { otherwise. }\end{cases}
$$


Then, define $\widehat{u}_{i^{\prime}}$ and $\widehat{w}_{k^{\prime}}$ as the payoffs of buyer $i^{\prime}$ and seller $k^{\prime}$ at assignment $\widehat{A}$, respectively. Then, by definition of $\widehat{A}$,

$$
\begin{aligned}
u_{i^{\prime}}+w_{k^{\prime}}-\left(\widehat{u}_{i^{\prime}}+\widehat{w}_{k^{\prime}}\right)=\left(v_{i^{\prime} j^{\prime}}-\Gamma_{i^{\prime} j^{\prime} k^{\prime \prime}}\right) \cdot A_{i^{\prime} j^{\prime} k^{\prime \prime}}+\left(v_{i^{\prime} j^{\prime}}-\Gamma_{i^{\prime} j^{\prime} k^{\prime}}\right) \cdot A_{i^{\prime} j^{\prime} k^{\prime}} & \quad+\left(\Gamma_{i^{\prime} j^{\prime} k^{\prime}}-r_{j^{\prime} k^{\prime}}\right) \cdot A_{i^{\prime} j^{\prime} k^{\prime}} \\
& \quad-\left(v_{i^{\prime} j^{\prime}}-\Gamma_{i^{\prime} j^{\prime} k^{\prime \prime}}\right) \cdot \widehat{A}_{i^{\prime} j^{\prime} k^{\prime \prime}}-\left(v_{i^{\prime} j^{\prime}}-\Gamma_{i^{\prime} j^{\prime} k^{\prime}}\right) \cdot \widehat{A}_{i^{\prime} j^{\prime} k^{\prime}} \\
& \quad-\left(\Gamma_{i^{\prime} j^{\prime} k^{\prime}}-r_{j^{\prime} k^{\prime}}\right) \cdot \widehat{A}_{i^{\prime} j^{\prime} k^{\prime}} \\
= & \left(v_{i^{\prime} j^{\prime}}-\Gamma_{i^{\prime} j^{\prime} k^{\prime \prime}}\right)-\left(v_{i^{\prime} j^{\prime}}-\Gamma_{i^{\prime} j^{\prime} k^{\prime}}\right)-\left(\Gamma_{i^{\prime} j^{\prime} k^{\prime}}-r_{j^{\prime} k^{\prime}}\right) \\
= & -\Gamma_{i^{\prime} j^{\prime} k^{\prime \prime}}+r_{j^{\prime} k^{\prime}} .
\end{aligned}
$$

Since $\left(i^{\prime}, k^{\prime \prime}\right) \in \mathcal{B}_{\alpha} \times \mathcal{S}_{\alpha}$ with $\alpha \in\{1,2\}$, we have that $\Gamma_{i^{\prime} j^{\prime} k^{\prime \prime}}=p_{j^{\prime}}$ and by assumption $p_{j^{\prime}}>r_{j^{\prime} k^{\prime}}, u_{i^{\prime}}+w_{k^{\prime}}-\left(\widehat{u}_{i^{\prime}}+\widehat{w}_{k^{\prime}}\right)<0$ contradicts (A.14). Assume now that for all $i^{\prime} \in \mathcal{B}_{1} \cup \mathcal{B}_{2}$ and all $k^{\prime \prime} \in \mathcal{S}_{1} \cup \mathcal{S}_{2}, A_{i^{\prime} j^{\prime} k^{\prime \prime}}=0$. By definition, $p_{j^{\prime}}=\min \left\{r_{j^{\prime} k} \mid k\right.$ is such that $\left.q_{j^{\prime} k}>0\right\}$. Let $k^{*} \in \mathcal{S}_{1} \cup \mathcal{S}_{2}$ be such that $q_{j^{\prime} k^{*}}>0$ and $p_{j^{\prime}}=r_{j^{\prime} k^{*}}$. By (1), such $k^{*}$ does exist. By (A.17) and the definition of $p_{j^{\prime}}, p_{j^{\prime}} \leq r_{j^{\prime} k^{\prime}}$, a contradiction with the initial assumption that $p_{j^{\prime}}>r_{j^{\prime} k^{\prime}}$.

Lemma 6 Let $M=(\mathcal{B}, \mathcal{G}, \mathcal{S}, V, d, R, Q)$ be a market and let $(u, w) \in \mathbb{R}^{B} \times \mathbb{R}^{S}$ be such that there exists a competitive equilibrium price vector $p$ such that for every coalition $\mathcal{C} \subset \mathcal{B} \cup \mathcal{S}$ and any feasible assignment $\widehat{A} S W$-compatible with $\mathcal{C}$ we have that

$$
\sum_{i \in \mathcal{B}^{\mathcal{C}}} u_{i}+\sum_{k \in \mathcal{S}^{\mathcal{C}}} w_{k} \geq \phi^{M}(\mathcal{C}, \widehat{A}, p) .
$$

Then, $(u, w) \in \mathcal{C E}$.

Proof Let $(u, w) \in \mathbb{R}^{B} \times \mathbb{R}^{S}$ be a payoff of market $M$ satisfying the hypothesis of Lemma 6 and let $A$ be any optimal assignment of market $M$. Consider first any coalition $\mathcal{C}=\{i\}$, where $i \in \mathcal{B}$. Observe that $A$ is itself $S W$-compatible with $\mathcal{C}$. Then, by assumption,

$$
u_{i} \geq \sum_{(j, k) \in \mathcal{G} \times \mathcal{S}}\left(v_{i j}-p_{j}\right) \cdot A_{i j k} .
$$

Consider now any coalition $\mathcal{C}=\{k\}$, where $k \in \mathcal{S}$. Observe that again $A$ is itself $S W$-compatible with $\mathcal{C}$. Then, by assumption,

$$
w_{k} \geq \sum_{(i, j) \in \mathcal{B} \times \mathcal{G}}\left(p_{j}-r_{j k}\right) \cdot A_{i j k} .
$$

Then,

$$
\sum_{i \in \mathcal{B}} u_{i}+\sum_{k \in \mathcal{S}} w_{k}=v(\mathcal{B} \cup \mathcal{S})=\sum_{(i, j, k) \in \mathcal{B} \times \mathcal{G} \times \mathcal{S}} \tau_{i j k} \cdot A_{i j k}
$$


By definition of the per unit gains $\tau_{i j k}$,

$$
\sum_{(i, j, k) \in \mathcal{B} \times \mathcal{G} \times \mathcal{S}} \tau_{i j k} \cdot A_{i j k}=\sum_{i \in \mathcal{B}} \sum_{(j, k) \in \mathcal{G} \times \mathcal{S}}\left(v_{i j}-p_{j}\right) \cdot A_{i j k}+\sum_{k \in \mathcal{S}} \sum_{(i, j) \in \mathcal{B} \times \mathcal{G}}\left(p_{j}-r_{j k}\right) \cdot A_{i j k}
$$

Hence, (A.18) and (A.19) imply that for every $i \in \mathcal{B}$ and $k \in \mathcal{S}$,

$$
\begin{aligned}
u_{i} & =\sum_{(j, k) \in \mathcal{G} \times \mathcal{S}}\left(v_{i j}-p_{j}\right) \cdot A_{i j k} \\
w_{k} & =\sum_{(i, j) \in \mathcal{B} \times \mathcal{G}}\left(p_{j}-r_{j k}\right) \cdot A_{i j k},
\end{aligned}
$$

and consequently, by Lemma 6 in Jaume, Massó, and Neme (2009), for every $i \in \mathcal{B}$ and $k \in \mathcal{S}$

$$
\begin{aligned}
u_{i} & =d_{i} \cdot \gamma_{i}(p) \geq 0 \\
w_{k} & =\sum_{j \in \mathcal{G}} q_{j k} \cdot \pi_{j k}(p) \geq 0 .
\end{aligned}
$$

Thus, $(u, w)=(u(p), w(p))$ and by $(10),(u, w) \in \mathcal{C} \mathcal{E}$.

Theorem 1 Let $(u, w),(\bar{u}, \bar{w}) \in \mathbb{R}_{+}^{B} \times \mathbb{R}_{+}^{S}$ be two payoff vectors of market $M=(\mathcal{B}, \mathcal{G}, \mathcal{S}, V, d, R, Q)$. Then,

$$
((u, w),(\bar{u}, \bar{w})) \in \mathcal{S} \mathcal{W}^{2 M} \text { if and only if }(u, w)=(\bar{u}, \bar{w}) \in \mathcal{C} \mathcal{E} .
$$

Proof Let $((u, w),(\bar{u}, \bar{w})) \in \mathcal{S} \mathcal{W}^{2 M}$. By Lemma 2, $(u, w)=(\bar{u}, \bar{w})$. By Lemma 5, there exists a competitive equilibrium price vector $p=\left(p_{1}, \ldots, p_{G}\right)$, with $p_{j} \geq \min \left\{r_{j k} \mid k \in \mathcal{S}\right.$ such that $\left.q_{j k}>0\right\}$ for all $j \in \mathcal{G}$, such that for any coalition $\mathcal{C} \subset \mathcal{B} \cup \mathcal{S}$, and any feasible assignment $\widehat{A}$ that is $S W$-compatible with $\mathcal{C}$, we have that:

$$
\sum_{i \in \mathcal{B}^{\mathcal{C}}} u_{i}+\sum_{k \in \mathcal{S}^{\mathcal{C}}} w_{k} \geq \phi^{M}(\mathcal{C}, \widehat{A}, p) .
$$

By Lemma $6,(u, w) \in \mathcal{C E}^{M}$.

Assume now that $(u, w) \in \mathcal{C} \mathcal{E}$. Then, $((u, w),(u, w)) \in \mathcal{C E}^{2 M}$. By Proposition $1,((u, w),(u, w)) \in$ $\mathcal{S} \mathcal{W}^{2 M}$.

Theorem 3 Let $\rho \in \mathbb{Z}_{+} \backslash\{0\}$. Then, there exist a market $M=(\mathcal{B}, \mathcal{G}, \mathcal{S}, V, d, R, Q)$ and a payoff vector $(u, w) \notin \mathcal{C E}$ such that $\left(u^{\rho}, w^{\rho}\right) \in \mathcal{C} o^{\rho M}$.

Proof Fix $\rho \in \mathbb{Z}_{+} \backslash\{0\}$. Define $M=(\mathcal{B}, \mathcal{G}, \mathcal{S}, V, d, R, Q)$ as follows: $\mathcal{B}=\left\{b_{1}\right\}, \mathcal{S}=$ $\left\{s_{1}, s_{2}\right\}, \mathcal{G}=\left\{g_{1}\right\}, v_{11}=1, r_{11}=r_{12}=0, d_{1}=4 \rho-1$ and $q_{11}=q_{12}=2 \rho$. It is easy to see 
that since the short side of the market is the demand, the unique competitive equilibrium price is $p_{1}=0$ and $\mathcal{C} \mathcal{E}=\{(4 \rho-1,0,0)\}$. Consider the payoff vector $(4 \rho-3,1,1) \notin \mathcal{C} \mathcal{E}$. We show that $\left((4 \rho-3)^{\rho},(1,1)^{\rho}\right) \in \mathcal{C} o^{\rho M}$. Let $\mathcal{C}$ be a coalition in market $\rho M$ with $\#\left(\bigcup_{\alpha=1}^{\rho} \mathcal{B}_{\alpha}^{\mathcal{C}}\right)=\beta$ and $\#\left(\bigcup_{\alpha=1}^{\rho} \mathcal{S}_{\alpha}^{\mathcal{C}}\right)=\sigma$. Thus,

$$
\beta \leq \rho \text { and } \sigma \leq 2 \rho
$$

The value of coalition $\mathcal{C}$ is

$$
v(\mathcal{C})= \begin{cases}\beta(4 \rho-1) & \text { if } 2 \beta \leq \sigma \\ 2 \rho \sigma & \text { if } 2 \beta>\sigma\end{cases}
$$

and

$$
\sum_{i \in \mathcal{B}^{\mathcal{C}}} u_{i}+\sum_{k \in \mathcal{S}^{\mathcal{C}}} w_{k}=\beta(4 \rho-3)+\sigma .
$$

We want to show that for all $\beta$ and $\sigma$ satisfying (A.20),

$$
\sum_{i \in \mathcal{B}^{\mathcal{C}}} u_{i}+\sum_{k \in \mathcal{S}^{\mathcal{C}}} w_{k} \geq v(\mathcal{C})
$$

Assume first that $\mathcal{C}$ is such that $2 \beta \leq \sigma$. Then, by (A.21) and (A.22), (A.23) holds if and only if $\beta(4 \rho-3)+\sigma \geq \beta(4 \rho-1)$ holds, which follows from $2 \beta \leq \sigma$.

Assume now that $\mathcal{C}$ is such that $2 \beta>\sigma$. Then, by (A.21) and (A.22), (A.23) holds if and only if $\beta(4 \rho-3)+\sigma \geq 2 \rho \sigma$ holds. Thus, to show that (A.23) holds is equivalent to show that

$$
\beta(4 \rho-3) \geq \sigma(2 \rho-1)
$$

holds. By (A.20), the most unfavorable case for which (A.24) holds is when $\sigma$ is larger; i.e., $\sigma=2 \gamma-1$. Hence, (A.24) follows if $\beta(4 \rho-3) \geq(2 \beta-1)(2 \rho-1)$. But this last inequality can be written as

$$
4 \beta \rho-3 \beta \geq 4 \beta \rho-2 \beta-2 \rho+1,
$$

which holds because $\beta \leq \rho$ and $\rho \geq 1$ imply $2 \rho-1 \geq \beta$. 